\title{
Type-I ELM precursor modes in JET
}

\author{
C.P. Perez ${ }^{1,2}$, H.R. Koslowski ${ }^{1}$, G.T.A. Huysmans ${ }^{3}$, T.C. Hender ${ }^{4}$, \\ P. Smeulders ${ }^{5}$, B. Alper ${ }^{4}$, E. de la Luna ${ }^{6}$, R.J. Hastie ${ }^{4}$, \\ L. Meneses ${ }^{7}$, M.F.F. Nave ${ }^{7}$, V. Parail ${ }^{4}$, M. Zerbini ${ }^{5}$ and JET-EFDA \\ Contributors $^{\mathrm{a}}$ \\ ${ }^{1}$ Institut für Plasmaphysik, Forschungszentrum Jülich, Trilateral Euregio Cluster, D-52425 \\ Jülich, Germany \\ ${ }^{2}$ FOM-Rijnhuizen, Ass. EURATOM-FOM, TEC, PO Box 1207, 3430 BE Nieuwegein, \\ Netherlands \\ ${ }^{3}$ Association Euratom-CEA, Cadarache, F-13108 St. Paul-lez-Durance, France \\ ${ }^{4}$ Euratom/UKAEA Fusion Association, Culham Science Centre, Abingdon, UK \\ 5 Associazione Euratom-ENEA, Centro Ricerche Frascati, Italy \\ ${ }^{6}$ Laboratorio Nacional de Fusión, Euratom-Ciemat, 28040 Madrid, Spain \\ ${ }^{7}$ Euratom/IST, Instituto Superior Tecnico, Lisbon, Portugal
}

Received 12 December 2002, accepted for publication 18 March 2004

Published 6 April 2004

Online at stacks.iop.org/NF/44/609

DOI: $10.1088 / 0029-5515 / 44 / 5 / 005$

\begin{abstract}
Clear low frequency (typically 5-25 kHz) coherent type-I ELM precursor modes have been identified in JET. They are detected through various diagnostics, especially in the ECE and Mirnov signals, but also on the SXR cameras and the multichannel O-mode edge reflectometer. The modes propagate in the direction of the ion-diamagnetic drift and are usually accompanied by a slight rise in the divertor $\mathrm{D}_{\alpha}$-emission. The precursors show no radial phase inversions and are localized to a few centimetres inside the separatrix, in the pedestal region. The time by which the precursor onset precedes the ELM event varies greatly, and ranges from $0.2 \mathrm{~ms}$ up to several tens of milliseconds, though the timescale has no effect on the characteristics of the precursors. During their lifetime, the precursor amplitude is often seen to fluctuate, and the frequency and dominant $n$-number of the precursors can change rapidly, typically within $1 \mathrm{~ms}$. The range of toroidal precursor mode numbers $n$ which have been observed is $1-13$. Parametric studies show that $n$ seems to be mainly prescribed by a combination of the electron pedestal pressure and the (normalized) electron pedestal collisionality $v_{\mathrm{e}}^{*}$, with increasing $v_{\mathrm{e}}^{*}$ leading to higher $n$-numbers. Above a certain edge collisionality (roughly $v_{\mathrm{e}}^{*}>1-3$ ) the precursors seem to be absent, indicating that a sufficiently high edge current is important to destabilize the precursors. The low- $n$ precursors are known to be external kinks (also known on JET as Outer Modes), while experimental findings and their comparison with stability calculations suggest that the precursor modes with higher $n$ are not pure external kinks but coupled ballooning-kink modes. Several hundred discharges have been analysed, and, in discharges with low to moderate edge collisionality, the precursors could be detected prior to most type-I ELMs, while prior to type-III ELMs the modes were not encountered. In spite of their regular occurrence, there is no evidence of the precursor mode growth rate rapidly accelerating before the ELM, indicating that type-I ELMs on JET are not triggered by these modes.
\end{abstract}

PACS numbers: 52.55.Tn, 52.35.Py

\section{Introduction}

Type-I ELMs [1-3] occur as quasiperiodic particle and heat losses near the plasma edge and are compatible with good confinement at high density. However, the energy losses associated with them are a concern for ITER due to the large transient heat loads expected on the divertor tiles [4,5]. Although their existence has been known for many years, their physics is not well understood, and the search for ELM

a See annex of Pamela J. et al 2003 Nucl. Fusion 431540. precursor activity which could shed light on its origin and control possibilities has been performed in many devices.

In counter-injected discharges in ASDEX-Upgrade, low frequency $(20 \mathrm{kHz})$ coherent oscillations starting $1 \mathrm{~ms}$ before the ELM have been detected by ECE channels resonant approximately $2 \mathrm{~cm}$ inside the separatrix, and by coils picking up oscillations of $B_{r}[6,7]$. Phase shift analysis using three toroidally distributed coils yielded $n=5-10$ [7]. No similar precursor activity could be observed in co-injected discharges. In a further report, density profile oscillations with $17 \mathrm{kHz}$ 
frequency in a counter-injected discharge were measured via reflectometry and associated with a type-I ELM precursor [8]. Later on, higher frequency $(70-150 \mathrm{kHz})$ precursors were reported in co-injected NBI discharges [9], detected with a newly installed enhanced Mirnov diagnostic. Up to three modes are observed at a time, and the toroidal mode numbers range from $n=3$ to 6 .

Magnetic oscillations with frequency $70-140 \mathrm{kHz}$ prior to type-I ELMs have been observed in Ohmic and ECRHheated discharges in COMPASS-D $[10,11]$ and, in the example presented, start at least $0.4 \mathrm{~ms}$ before the ELM. They propagate in the direction of the electron diamagnetic drift and are often composed of two beating modes. From the ratio of the plasma rotation to the mode frequencies, the toroidal mode numbers were determined as $n=4$ and 5 .

On DIII-D a general broadband fluctuation level increasing $30-50 \mathrm{~ms}$ before type-I ELMs is seen, but no coherent magnetic precursors seem to be observed [12]. Occasionally, some burst-like density fluctuations are seen [13].

On JT-60U, coherent density fluctuations near the plasma edge with $10-25 \mathrm{kHz}$ frequency could be measured with reflectometry [14]. They are observed prior to most type-I ELMs and start approximately 200-500 $\mu$ s before the ELM. The oscillations could not be detected on the magnetics.

In JET low frequency modes $(\sim 15 \mathrm{kHz})$ preceding giant ELMs $^{1}$ have been reported in [15]. They are observed within a few milliseconds of the $\mathrm{D}_{\alpha}$ spike and have $n=2$ and 4 in the example shown. Longer lived precursors at higher frequency $(f \sim 80 \mathrm{kHz})$ and with high toroidal mode numbers $(n=3-12)$ are also observed $[15,16]$. Although in [16] it is not explicitly stated whether the ELMs analysed are type-III or type-I/giant ones, this work is often cited in the context of type-III ELMs. However, figure 4 of [16] is devoted to a giant ELM, namely the same as figure 8 of [15].

In hot-ion H-mode discharges [17] giant ELMs were generally preceded by so-called Outer Modes (OMs) [18], although they have not been explicitly labelled as precursors. Having low mode numbers ( $n$ mostly 1 or 2 ) and frequency ( $f \sim 5-15 \mathrm{kHz}$ ), they could be very long lived, occasionally existing for up to hundreds of milliseconds. OMs have been identified as low- $n$ external kinks [19].

Subsequent to the JET references cited above several improvements to diagnostics, data acquisition and MHD analysis codes were made. In this paper, we will present and study in detail precursor activity found prior to type-I ELMs in general (not restricted to giant ones) on JET. The term 'precursor' is used here in its more general sense to denote modes that systematically precede ELMs, while the term 'trigger' is used to denote those modes whose growth rate rapidly accelerates prior to the ELM. ELM triggers are also ELM precursors, but ELM precursors do not necessarily have to be ELM triggers. The outline of this paper is as follows: section 2 is devoted to the experimental observations for a class of lower frequency precursors observed, and the properties of lower- and higher- $n$ precursors will be compared in section 3 . Section 4 gives an overview of the ideal MHD activity that, according to modelling calculations, can be expected at the

\footnotetext{
1 The term 'giant' has not acquired a standardized meaning in the literature. Following [15] it is used at JET to denote bursts of $\mathrm{D}_{\alpha}$ above a certain amplitude.
}

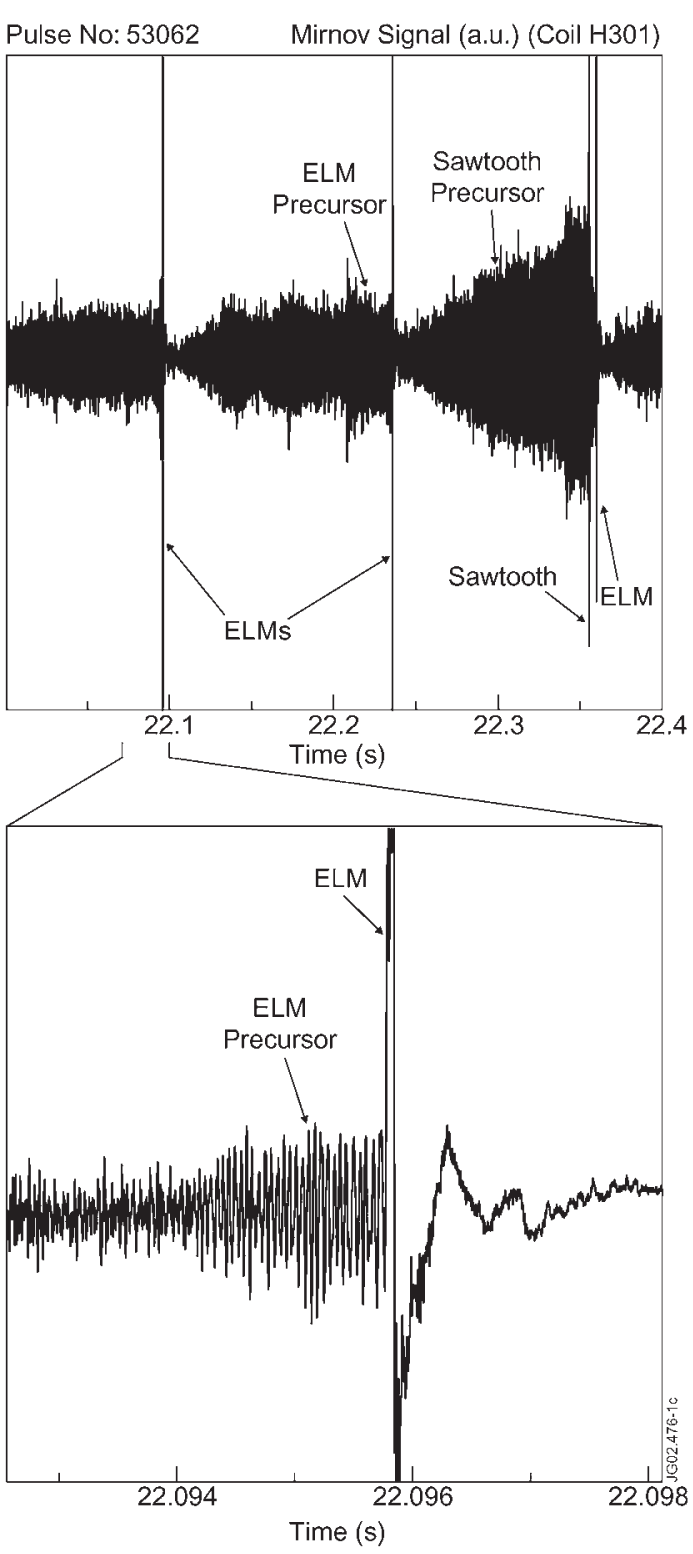

Figure 1. Mirnov signals of a coil located at the low-field side for discharge 53062. The parameters of this discharge at the relevant time were: $B=2.7 \mathrm{~T}, I=2.5 \mathrm{MA}, P_{\mathrm{NBI}}=13.2 \mathrm{MW}$ (co-injected), $\bar{n}_{\mathrm{e}}=7 \times 10^{19} \mathrm{~m}^{-3}, \kappa=1.75, \delta_{u / l}=0.36 / 0.24$.

plasma edge, and compares it to the experimental findings. Finally, a discussion of the results will be presented in section 5 .

\section{Experimental observations}

\subsection{Magnetic measurements}

In the time window of figure 1 three type-I ELMs and a sawtooth crash occur. The first ELM is preceded by a shorter precursor $(\sim 2 \mathrm{~ms})$, shown in the zoom view, while the second and third ELMs are preceded by more long-lived precursors ( $\sim 30 \mathrm{~ms}$ ), which are difficult to see here. Compared to other MHD activity like internal kinks or NTMs, the precursors are much weaker and often difficult to observe directly on the Mirnov signals. Figure 2 shows the corresponding spectrogram, where the three precursors $(f \sim 18 \mathrm{kHz})$ are 


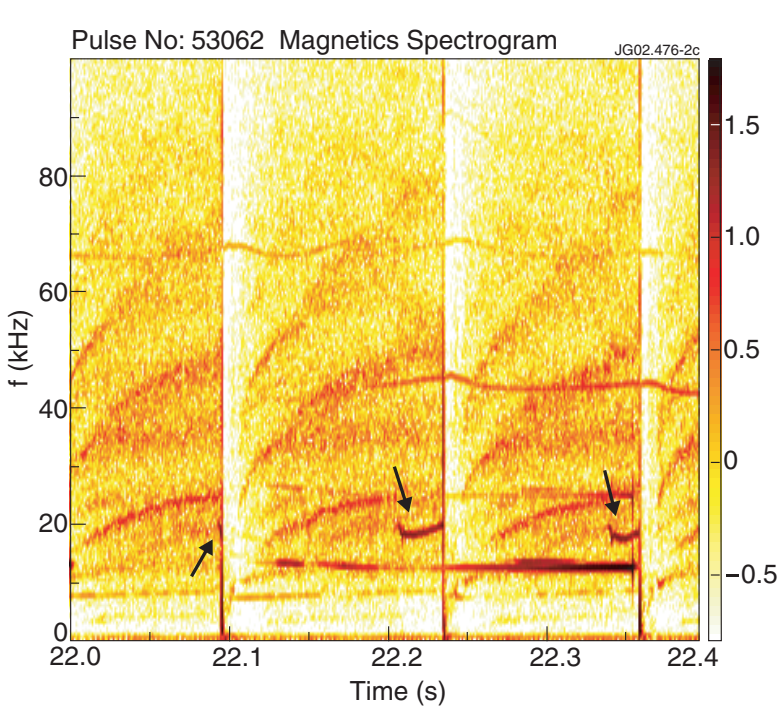

Figure 2. Magnetics spectrogram from a coil located on the low-field side, with the precursors marked by the arrows. The stronger mode at $13 \mathrm{kHz}$ is the sawtooth precursor. The numbers to the right of the colourscale denote the logarithm $\left(\log _{10}\right)$ of amplitude, and are given in a.u.

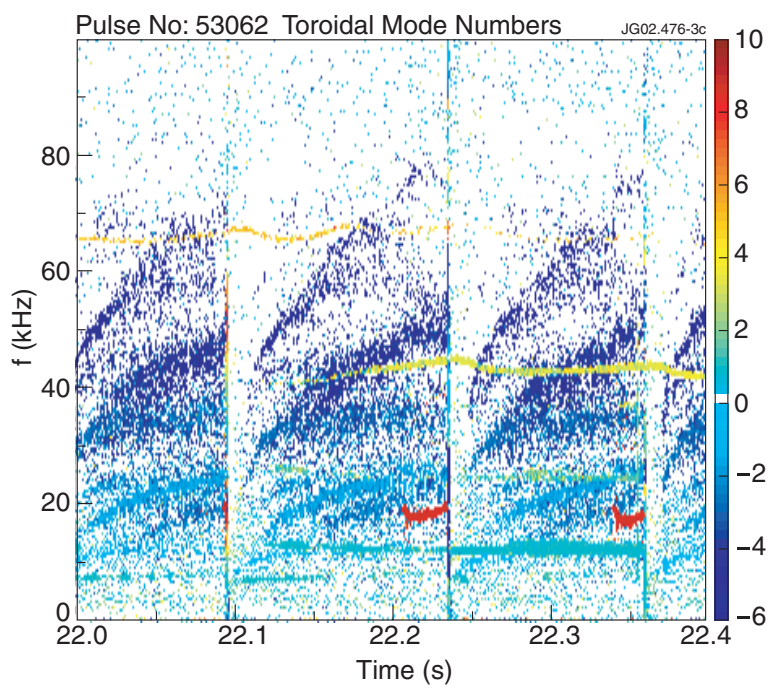

Figure 3. Spectrum of toroidal mode numbers $n$. For the three ELMs shown the precursors have $n=8$ (red).

marked by arrows. The first precursor can hardly be seen due to its short life. At their usual frequencies precursors shorter than $1 \mathrm{~ms}$ are difficult, or impossible, to discern on spectrograms due to the finite number of sampling points required for the Fourier analysis.

The toroidal mode numbers can be inferred from mode number spectra as shown in figure 3 , where the colours denote the toroidal mode numbers $n$. By making a Fourier decomposition of a toroidal set of Mirnov coils and analysing the phase shift of the fluctuations the $n$-numbers can be obtained. For the analysis a high resolution array of five coils, with toroidal angles $\Delta \phi$ gradually increasing from $1.7^{\circ}$ to $15.9^{\circ}$, was used, adequate for mode numbers $n \leqslant 11$. A subset of the coils with lower $\Delta \phi$ was employed if higher mode numbers needed to be resolved or simply to check the correctness of previous calculations. The coils are positioned close to the plasma boundary, with $r_{\text {coil }} / r_{\text {sep }}$ being roughly 1.2 , depending on the plasma shape. It proves very useful for finding hidden activity to plot the mode numbers in a spectrogram-like way due to its twofold filtering: in frequencyand in phase-space. This increases the contrast and makes it possible to discern modes faintly visible on the spectrograms. To reduce the noise level of the plots, points are discarded when the amplitudes are below a user-defined threshold or the fitting error of the mode number exceeds a certain amount. Positive and negative mode numbers are detected. Modes with negative $n$ rotate in the opposite direction to modes with positive $n$. The convention used here is that modes with negative $n$ rotate in the direction of the electron diamagnetic drift. For the three ELMs shown the precursors have $n=+8$, and similar frequencies, despite their different duration. The range of toroidal precursor mode numbers that have been observed is 1-13. Precursors with $n>11$ are seen rather seldom, and precursors with $n>13$ seem to not occur at all. The upper limit of observed precursor $n$-numbers is most likely physically real and not imposed by a diagnostic limitation. Although the radial damping rate of magnetic perturbations increases with increasing mode numbers, even precursors with very high $n \sim 11-13$ could still be clearly discerned above the noise level on Mirnov signals. Therefore, it should have been possible to detect eventually occurring precursors with slightly higher $n \gtrsim 13$ on the magnetics if they had been present. As noted above, the toroidal spacing of the coils used is easily capable of resolving higher toroidal mode numbers, and, for example, other edge MHD activity with $n=15-20$ has been detected.

In addition, one observes in figure 3 several broader bands of magnetic fluctuation with higher frequency ( $f=10-80 \mathrm{kHz}$ ) propagating in the direction of the electron diamagnetic drift (negative $n$ ), with $n$ typically ranging from -1 to -8 . These bands were already studied in [20] and named washboard modes. Washboard modes are plasma edge instabilities, and neither their origin nor their driving force is known. Resistive ballooning modes have been discussed as a possible candidate to explain their origin in [20]. The physics of washboard modes is beyond the scope of this paper, but, without going into much detail, it is worth noting that washboard modes and ELM precursors interact, with the ELM precursors tending to inhibit the washboard modes. The physical mechanism behind this interaction has not yet been identified. For a detailed description of washboard mode dynamics and their consequences for the plasma edge the reader is referred to [21].

From the above example it could be seen that the time by which the precursor onset precedes the ELM event, and hence the precursor lifetime, varies greatly. The observed precursor lifetimes range from $0.2 \mathrm{~ms}$ up to several tens of milliseconds, or even longer. Low- $n$ precursors generally start at least 10 or $20 \mathrm{~ms}$ prior to the ELM. Some cases have been observed where they start as early as a few hundred milliseconds prior to the ELM. High- $n$ precursors tend to be more short lived. They start typically a few milliseconds up to a few tens of milliseconds before the ELM. Since long-lived precursors are easier to study with the diagnostics and have all other characteristics similar to short-lived precursors, we will use them often throughout this paper.

The analysis of the poloidal mode numbers $m$ on the basis of poloidally distributed coils is more complicated. Due to 
Table 1. Values for the poloidal mode numbers $m$ of ELM precursors with $n=1$, obtained from the analysis of the phase shifts of fluctuations measured by four poloidally distributed Mirnov coils. The true $m$-numbers of the modes must be integer numbers. Also given for each discharge is the safety factor at $95 \%$ of the poloidal flux, $q_{95}$, calculated by EFIT.

\begin{tabular}{llll}
\hline Pulse no. & $n$-number & $m$-number & $q_{95}$ \\
\hline 42578 & 1 & $4.3-4.8$ & 3.8 \\
42840 & 1 & $4.0-5.2$ & 3.9 \\
42870 & 1 & $3.4-3.8$ & 3.8 \\
43011 & 1 & $4.3-5.0$ & 3.8 \\
43021 & 1 & $3.5-4.0$ & 3.4 \\
43603 & 1 & $3.8-4.3$ & 3.3 \\
52010 & 1 & $3.3-3.8$ & 2.9 \\
\hline
\end{tabular}

the plasma toroidicity the apparent $m$-number depends on the poloidal position of the coils (the so-called $\theta^{*}$-effect $[22,23]$ ). Plasma shaping (elongation, triangularity) and the presence of an X-point impose further difficulties for modes located close to the plasma boundary. The X-point, in particular, can distort the poloidal mode structure considerably. Poloidal mode numbers determined by coils picking up magnetic fluctuations around the plasma midplane $(\theta=0, \pi)$ will then have the tendency to be lower than the real $m$-number of the mode. Only a limited numbers of coils, distributed poloidally around the plasma cross section, are available on JET. Four coils are used for the analysis, two on the low-field side ( $6^{\circ}$ apart), and two on the high-field side (50 apart). Due to aliasing effects the set of coils is not suitable for the analysis of modes with high $m$-numbers, but for lower $m(\lesssim 6)$ it has proved to be reasonably reliable for modes such as NTMs [24]. The procedure used for the analysis is described in more detail in appendix B of [20]. For the ELM precursors with the lowest $n(n=1)$ the poloidal mode number analysis gives reproducible results, as summarized in table 1. For each discharge, one to three ELM precursors have been analysed. The resulting $m$-numbers were not found to change significantly within each discharge. The $m$-numbers obtained are comparable to, or slightly larger than, $q_{95}$, and thus correspond to rational surfaces located close to the plasma boundary.

\subsection{Mode location and structure}

The radial location of the precursors can be most easily determined through ECE measurements. JET's ECE system consists of 48 heterodyne radiometers sampled with $250 \mathrm{kHz}$, measuring slightly below the plasma midplane. The spacing between resonant measurement radii is typically only 1 or $2 \mathrm{~cm}$. The precursors always occur near the separatrix, in the pedestal region. Figure 4 shows a set of edge ECE signals for our previous example, and figure 5 shows the respective ECE profile and the location of the separatrix, with an uncertainty of approximately $1 \mathrm{~cm}$, as calculated by the EFIT equilibrium code. The channels at $a-5.0 \mathrm{~cm}$ and $a-3.9 \mathrm{~cm}$ measure near the top of the pedestal ( $a=$ separatrix radius). The oscillations are most clearly seen $2.6 \mathrm{~cm}$ inside the separatrix, well inside the optically thick region, enhanced by the large temperature gradients in the pedestal. The temperature oscillations of the two long-lived precursors prior to the second and the third ELM grow within $2 \mathrm{~ms}$ and then saturate in amplitude until $2 \mathrm{~ms}$ and $0.7 \mathrm{~ms}$ before the ELM, respectively. Then,

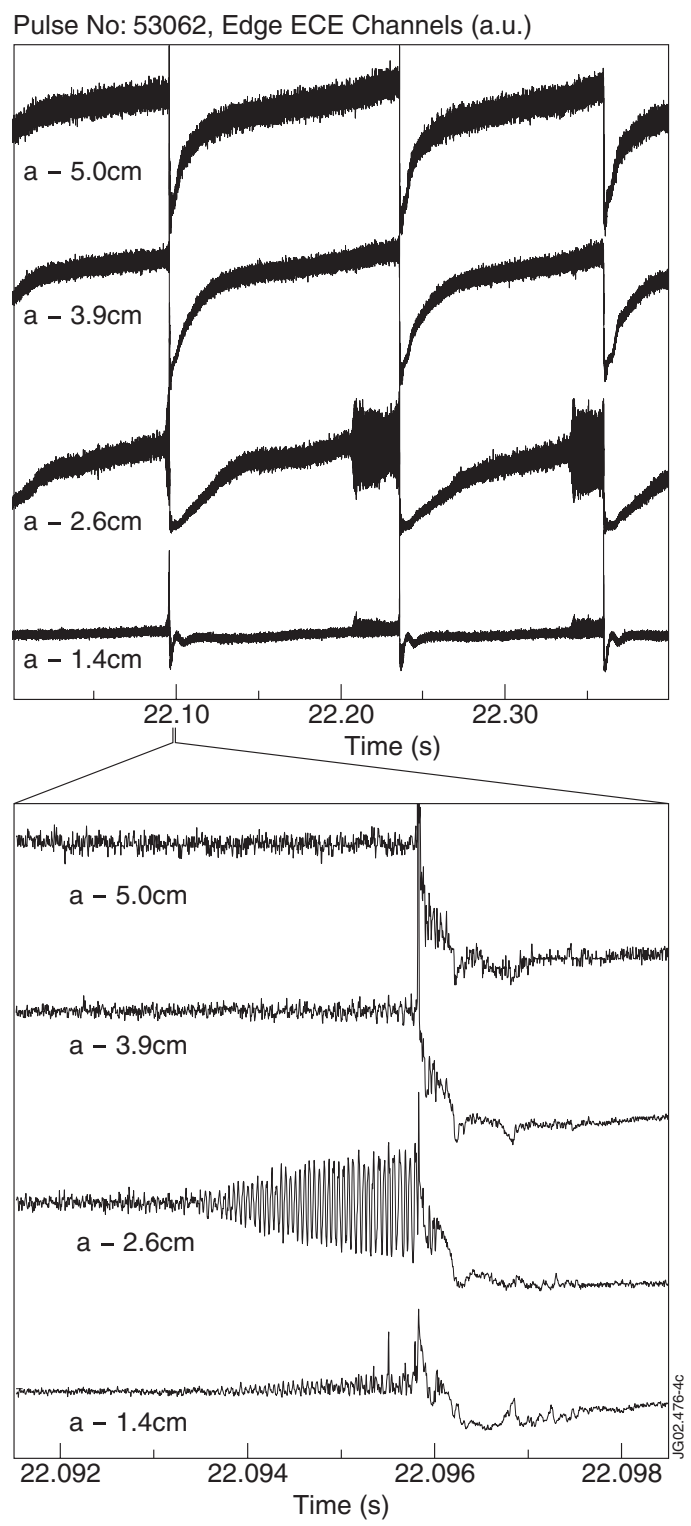

Figure 4. Edge ECE signals together with the distance of the measurement radii to the separatrix as calculated by EFIT. The second picture shows a zoom of the shorter precursor prior to the first ELM.

they begin to grow again, albeit with growth rates that are much smaller than in the first growth phase. The precursor prior to the first ELM is interrupted by the ELM already during the initial growth phase of the oscillations. In all three cases the temperature oscillations grow only roughly linearly in time when approaching the crash.

Concerning ECE channels further outwards towards the separatrix one has to be cautious. When the density becomes too low, the plasma does not behave as a black body, and the radiation then detected at a given frequency can have contributions from different plasma regions (shine-through effect). The interpretation of the signal using a local model is then impossible. This shows up in the ECE profile, where the level of emission picked up artificially rises again (dotted part of the profile). Another complicating factor is that while it is difficult to locate the origin of the shine-through radiation 


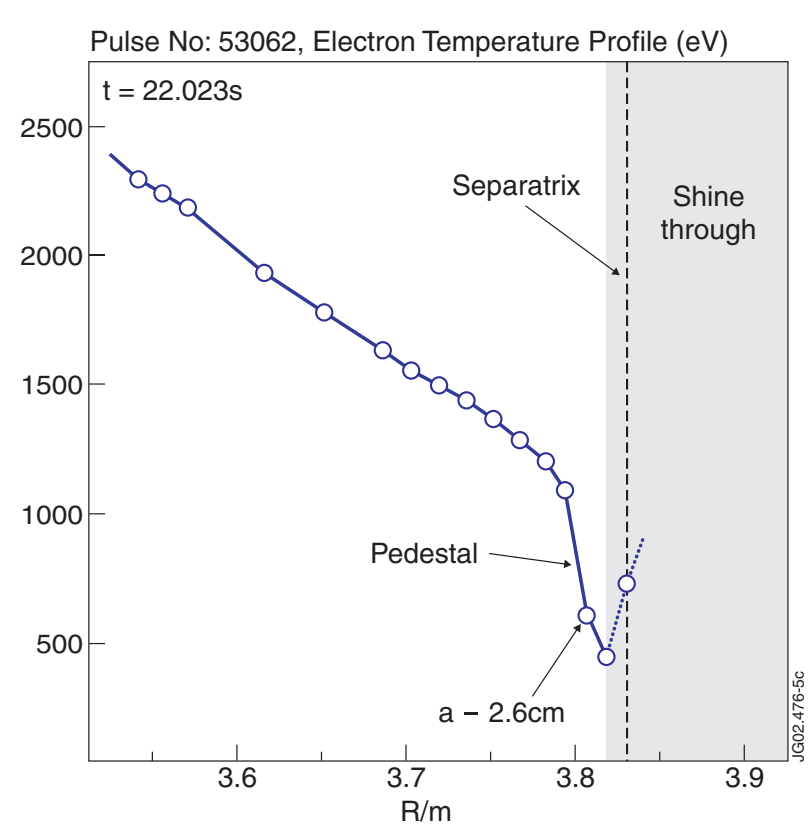

Figure 5. Electron temperature profile as measured by the ECE. The arrow marks the channel where the signal oscillations are most prominent in figure 4, supported by the large temperature gradients in the pedestal. The shine-through effect impedes a full coverage of the measurements out to the separatrix.

picked up, the amount of shine-through itself is additionally modulated by the density oscillations caused locally by the mode, which reflectometry confirms to be present. The optical thickness $\tau$ has been evaluated in the cold plasma approximation (the formulae are given in [25], table 4) for the channels resonant at $a-2.6 \mathrm{~cm}$ and $a-1.4 \mathrm{~cm}$. The values obtained for $\tau$ strongly depend on the assumed shape of the edge density profile. At $a-2.6 \mathrm{~cm}, \tau=4-11$, while at $a-1.4 \mathrm{~cm}, \tau=1.3-3.9$. For optical thickness $\tau>2$ is required. The marginal channel at $a-1.4 \mathrm{~cm}$ is thus dubious and should be disregarded. Hence, only ECE data from channels measuring at optically thick radii are taken into account.

Figure 6 shows results from the coherence analysis of a Mirnov signal with the edge ECE signals. The colour in the first two plots depicts the value of the coherence and the phase of the cross spectral density, respectively, as a function of frequency and distance to the separatrix. The coherence value increases towards the edge, and the phase shows no inversions across the radii, suggesting a kink-like mode, although this data could be produced as well by a magnetic island located between the last optically thick ECE channel and the separatrix. The values of the radial displacement shown in figure 6 are found to increase rather sharply at the edge, with displacements of the order of $1 \mathrm{~cm}$. One can further see that for our $n=8$ precursor the mode extent is not limited to $a-2.6 \mathrm{~cm}$, where the oscillations were clearly visible in figure 4 . Large displacements also occur one channel further inwards, measuring at the pedestal shoulder, where the modest temperature gradient made them more difficult to observe directly on the ECE signal. The method used to obtain the displacement from the coherence analysis is described in appendix $C$ of [20]. In general, the displacements observed typically range from $1 \mathrm{~mm}$ up to $1.5 \mathrm{~cm}$. The mode location is further verified by an edge reflectometer. JET's
O-mode reflectometer system consists of 10 channels with cut-off densities ranging from 6.0 down to $0.43 \times 10^{19} \mathrm{~m}^{-3}$, viewing the plasma from the low-field side, near the plasma midplane. In general, the modes are seen on all channels or a subset of channels with lower cut-off densities, depending on the discharge density. The fact that the modes are seen on the channels with low cut-off densities, measuring at pedestal radii that normally include radii where the ECE suffers from shine-through, indicates that the precursors indeed extend from about the pedestal shoulder, as seen from ECE measurements, until at least fairly close to the separatrix. Figure 7 shows the fringe-jump corrected traces for an $n=8$ precursor together with the distance of their reflecting layers to the separatrix. Since we lack the wherewithal to measure fast density profiles at the edge, we have estimated the cut-off radii by taking the line-averaged density of the edge interferometer cord to be the density at the top of the pedestal, and then linearly extrapolate to zero density at the separatrix. Allowing for a finite density in the SOL would move the radii of the channels with lowest cutoff density further outwards, possibly outside the separatrix. The oscillations show no phase inversions between channels, confirming that the mode has a kink-like radial structure, i.e. twisting parity, and is not a magnetic island.

With the inserted filters very little information could be obtained from the SXR cameras due to their low response at the plasma boundary and the line-averaging. Only strong enough precursors could be faintly detected through spectrograms of channels looking towards the plasma edge, consistent with a mode localized in the plasma periphery. For a camera viewing the plasma laterally from the low-field side, comparison of channels looking towards the top and bottom of the plasma (tangentially towards the same flux surfaces) yielded no evident up-down amplitude asymmetry.

Finally, the precursor occurrence is normally accompanied by a slight increase of $\mathrm{D}_{\alpha}$ emission (figure 8). The increased $D_{\alpha}$ could arise from a small increase in particle and/or energy transport across the pedestal associated with the precursors.

\subsection{Further properties}

The precursors are variable in the sense that they might grow and shrink repeatedly before the ELM or change their dominant $n$-numbers and frequency rapidly, at first sight, without an apparent cause, that is, with no major changes in the main plasma parameters. Figure 9 shows an example for the change in amplitude, where the $n$-spectrum and ECE signals show long intermittent $n=7$ (orange) precursors at 10-15 $\mathrm{kHz}$ prior to two type-I ELMs at 21.1 and $21.34 \mathrm{~s}$. Between 21.0-21.1 s and $21.2-21.35 \mathrm{~s}$ the plasma current increased marginally from 2.49 to $2.53 \mathrm{MA}$, recovering the current lost by the preceding ELM, but whether this is really responsible for the mode behaviour in this particular case remains unclear. One can see that this behaviour is restricted to the plasma edge; the core MHD activity (e.g. a sawtooth precursor at $12 \mathrm{kHz}$ and a $4 / 3$ mode around $32 \mathrm{kHz}$ ) remains unperturbed. At $\sim 45 \mathrm{kHz}$ one can observe a further mode with similar frequency behaviour and higher $n, \geqslant 10$ (for modes with higher mode numbers than given in the scale, the colour of the mode saturates at the highest colour of the scale). A separate analysis with a reduced set of coils to resolve higher mode numbers yielded $n=11-13$. 

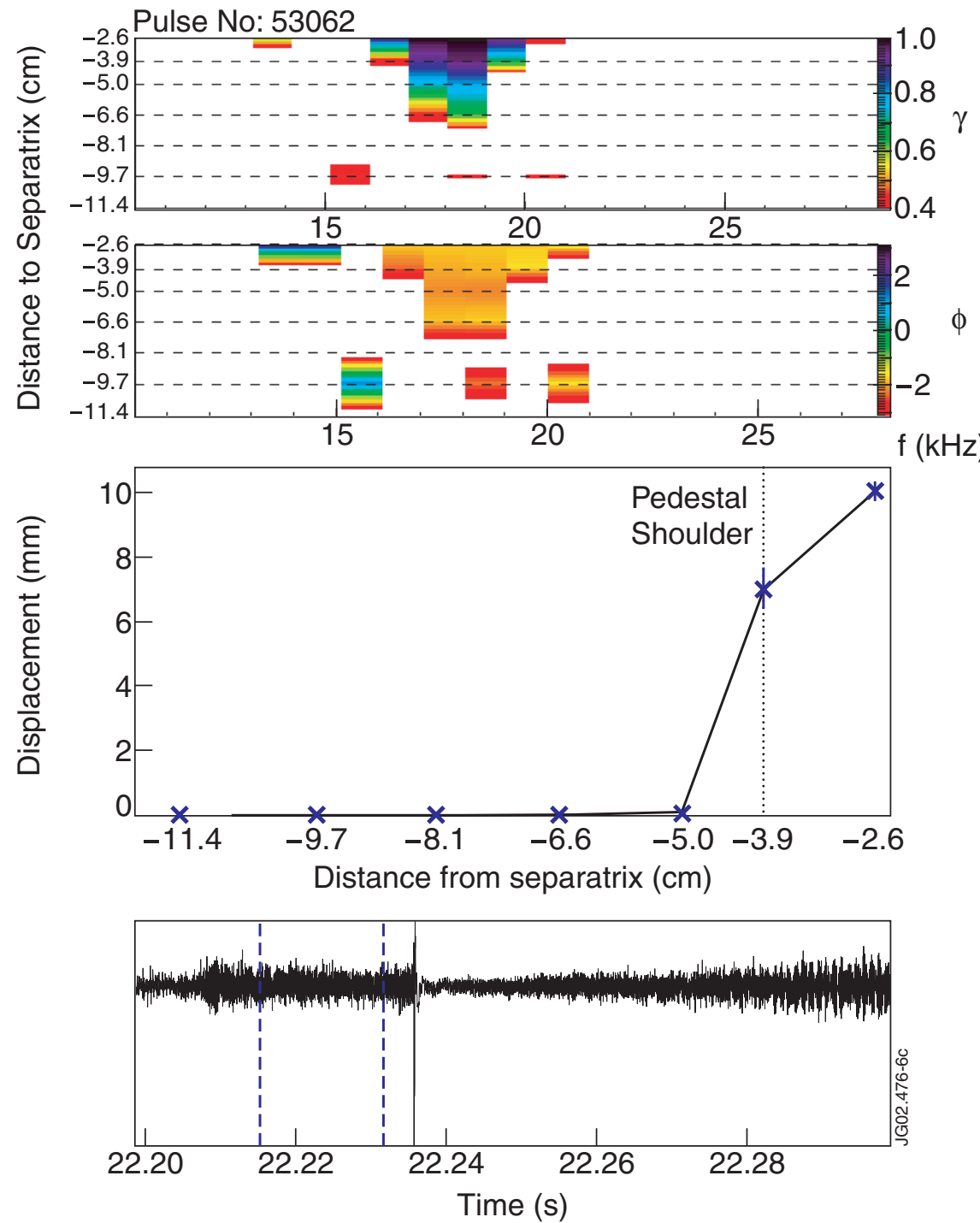

Figure 6. Coherence analysis of a Mirnov coil with the edge ECE channels. The first two plots give the values of the coherence and of the phase of the cross-spectral density, respectively, as a function of frequency and distance to the separatrix. The phase shows no variations with radius, suggesting a kink-like mode. The third window shows the magnitude of the radial mode displacement as a function of radius, and the fourth one shows the Mirnov signal with the time interval used for the coherence analysis (within the dashed blue lines).

Figure 10 shows an example in which the dominant precursor mode numbers change spontaneously within approximately $500 \mu \mathrm{s}$ : starting with $n=9(15 \mathrm{kHz})$, the dominant precursor becomes $n=7(8 \mathrm{kHz})$ and finally $n=5$ $(\sim 1 \mathrm{kHz})$. In spite of being observed at similar radii, the observed precursor frequencies are not proportional to their $n$-numbers. Considering the limited spatial resolution of the ECE measurements, the most likely explanation is that the various precursors differ slightly in their mode location and structure, and that this might indeed play a role in the final propagation frequency in a plasma region where changes in gradients and/or rotation velocities are expected to occur on a very short scale length.

Sometimes it happens that multiple precursors precede an ELM. An example is shown in figure 11 where a whole range of modes could be detected. One can distinguish precursors with $n=6(\sim 9 \mathrm{kHz}), n=7$ (the very long-lived mode around $\sim 13 \mathrm{kHz}), n=8(\sim 18 \mathrm{kHz})$ and at slightly higher frequencies further modes with $n=9$ and 10 . Around $50 \mathrm{kHz}$ one sees a further very long-lasting mode with unusually high mode number and frequency, for which a separate analysis with a reduced set of coils yielded $n=11-13$. Further analysis revealed that the $n=7$ mode dominates over a long time, but becomes weaker shortly before the ELM. Simultaneously, the $n=8$ mode becomes dominant until, finally, the ELM occurs. The other modes turn out to be much weaker.

In general, the observations described above suggest that there is a whole pool of mode numbers available to the ELM precursors, which can be explained by the closeness of rational surfaces due to the high magnetic shear near the separatrix. It seems that some minor local parameter modifications in the plasma periphery are sufficient to stabilize or destabilize individual modes and to change their frequency or the most unstable mode numbers.

It is difficult to say whether the precursors interact or not. Several cases like the one above have been observed where the 

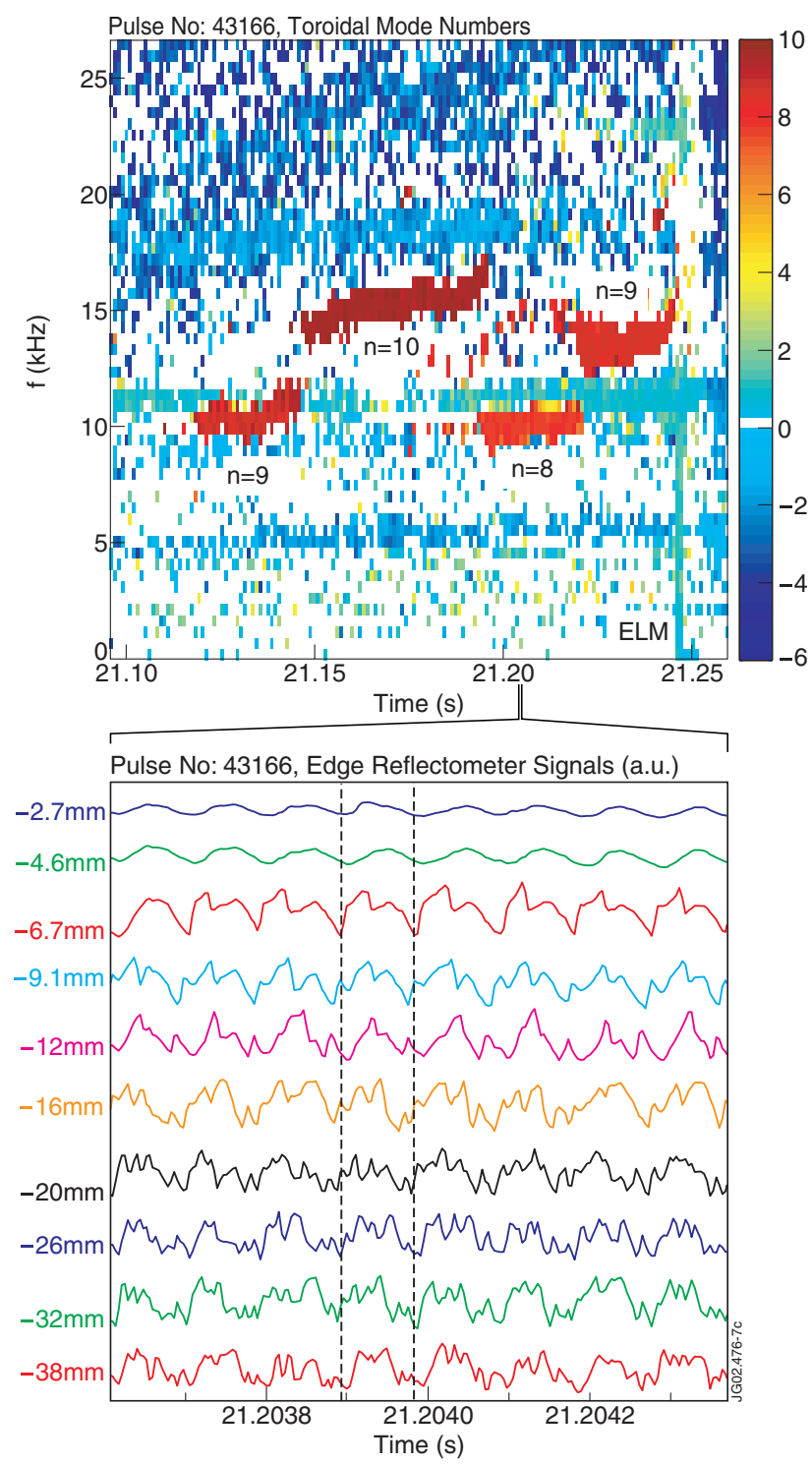

Figure 7. Toroidal mode numbers showing a sequence of precursors preceding an ELM, and traces of the ten edge reflectometer channels showing the oscillations corresponding to the $n=8$ precursor. Together with the traces, the estimated radii of the reflecting layers relative to the separatrix radius are given. The cut-off densities range from $0.43 \times 10^{19} \mathrm{~m}^{-3}$ (uppermost channel) to $6.0 \times 10^{19} \mathrm{~m}^{-3}$ (lowermost channel). No phase inversions are observed across the radii. The dashed lines are to guide the eye.

weakening of one precursor was followed by the appearance or growth of another one, as if the modes were competing amongst themselves trying to inhibit each other. Alternatively, this could again be explained by spontaneous minor radial shifts of the mode rational surfaces, always possible as the current magnitude and distribution are not ideally constant, with respect to the radii with largest driving forces, namely pressure and/or current gradients. On the other hand, cases have been found where at least two precursors with comparable amplitude coexist with no signs of interaction. In any case, the present data do not suggest that ELMs originate from a nonlinear coupling or mutual reinforcement of this kind of precursors.
Pulse No: $53062, \mathrm{D}_{\alpha}\left(10^{15} \mathrm{ph} / \mathrm{s} / \mathrm{sr} / \mathrm{cm}^{2}\right)$

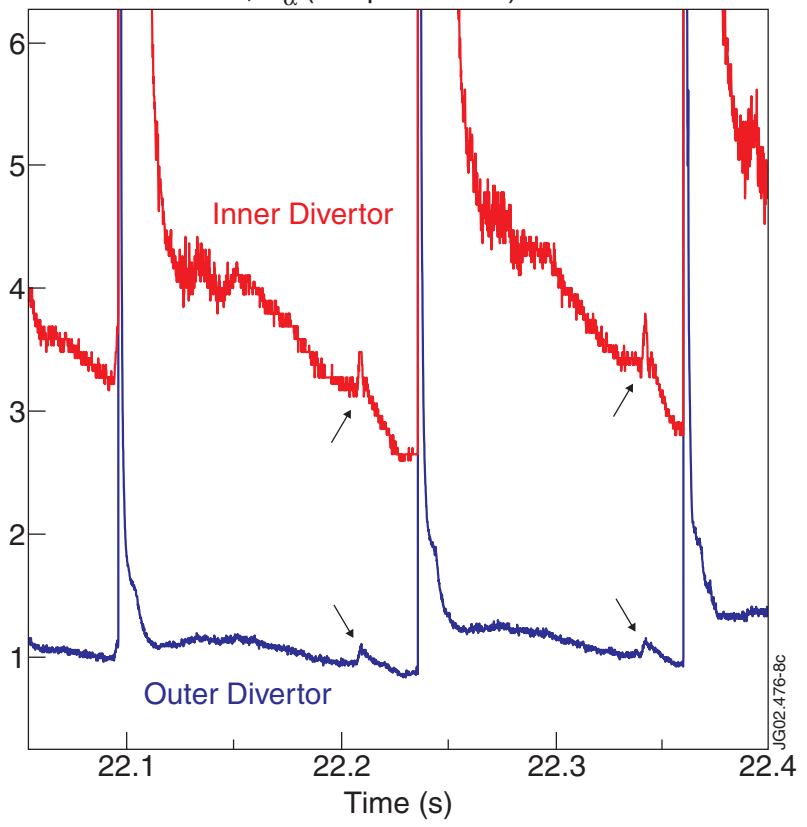

Figure 8. Inner and outer divertor $\mathrm{D}_{\alpha}$ signals for the example of figure 2. The arrows point to the time of the precursor onset.

\section{Comparison of low- and high-n modes}

In the previous section it has been mentioned that the precursor mode numbers cover a wide range from $n=1-13$. Precursors with low $n=1-3$ were regularly observed in the so-called hot-ion H-mode discharges [17], and are seen in some conventional ELMy H-mode discharges as well. Hot-ion $\mathrm{H}$-modes are characterized by very high ion temperatures achieved by applying NBI heating to a low density target. The low- $n$ modes occurring in them were not explicitly labelled as ELM precursors, and have been named OMs [18] due to their localization at the plasma boundary. The situation is that not all OMs ended with an ELM, but ELMs were generally preceded by OMs. By analysing the phase and amplitude behaviour of the oscillations detected on multiple SXR channels and comparing them to modelling results, OMs have been identified as low- $n$ external kinks [19]. Although there is a priori no sharp boundary in the transition from low to high $n$-numbers that would suggest the higher- $n$ precursors to be of a different nature, strictly speaking, only the low- $n$ modes are experimentally known to be external kinks. This makes a comparison of low- $n$ with intermediateand higher- $n$ precursors particularly interesting. It was not possible, however, to repeat the analysis procedure of [19] for higher- $n$ precursors. Many of the SXR diodes have suffered radiation damage in the $\mathrm{D}-\mathrm{T}$ campaign, and the precursor oscillations in the remaining SXR channels were too faint (in contrast to the OM cases used by that time, which benefited from very high edge temperatures).

\subsection{Low-n precursors}

As an example of low- $n$ precursors, two giant ELMs with their respective OMs are depicted in figure 12, including a Mirnov signal and its respective spectrogram, as well as 

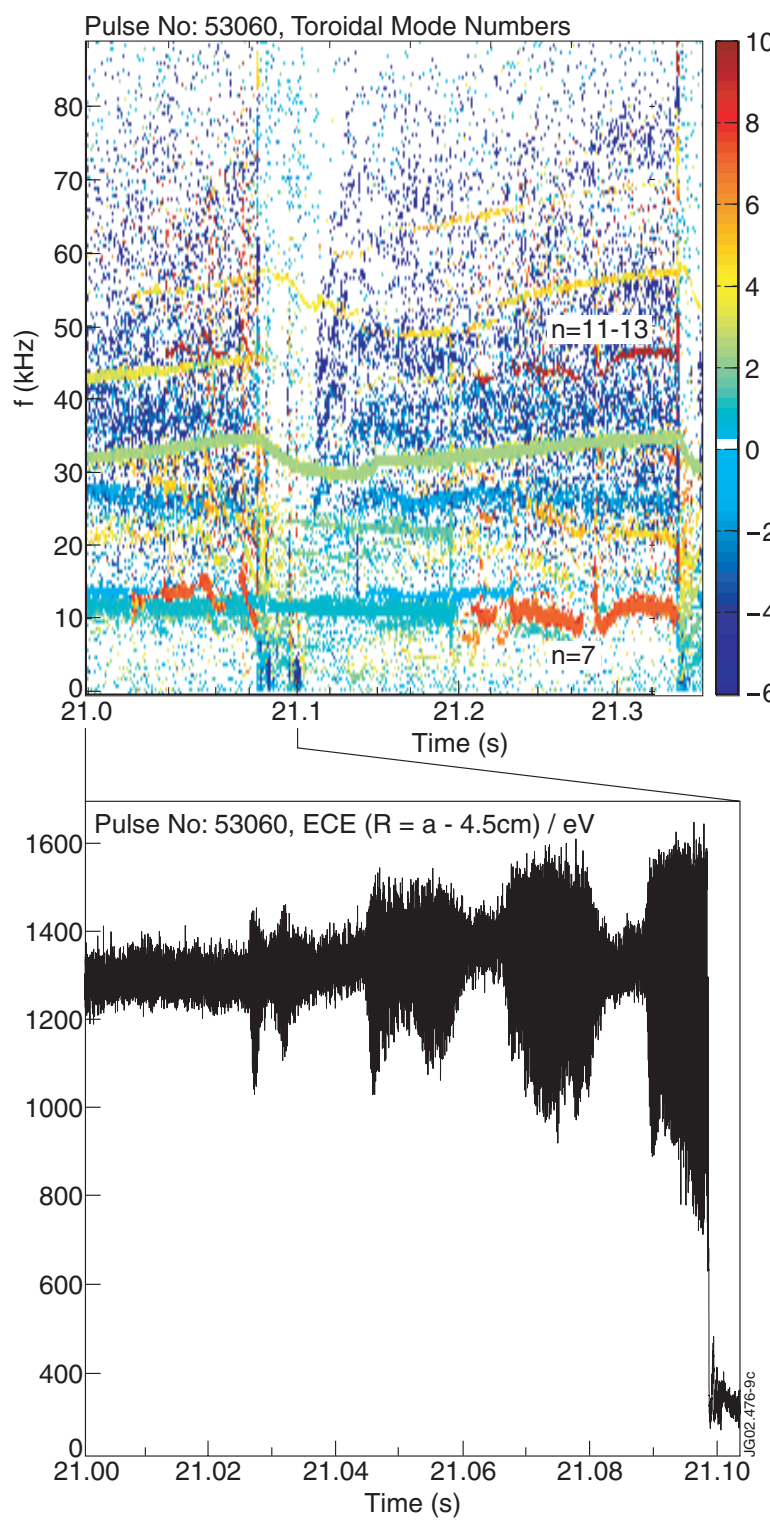

Figure 9. Toroidal mode numbers and ECE emission $4.5 \mathrm{~cm}$ inside the separatrix (near the pedestal top) showing intermittent $n=7$ (orange coloured) precursor modes at $10-15 \mathrm{kHz}$ prior to two type-I ELMs at roughly $21.1 \mathrm{~s}$ and $21.34 \mathrm{~s}$, respectively.

the spectrum of toroidal mode numbers. The precursors are composed of a dominant $n=1$ component with up to ten or twelve harmonics, which is usually regarded as a sign of high (poloidal or toroidal) localization. It has been observed that with increasing precursor $n$-numbers the number of harmonics decreases systematically. For instance, precursors with dominant $n=2$ are usually composed of four or five harmonics, $n=4$ precursors have mostly an $n=8$ harmonic, and precursors with $n \geqslant 5$ usually do not show additional harmonics. Linked to this, the signal oscillations associated with lower- $n$ precursors are, compared to higher- $n$ precursors, much less sinusoidal.

\subsection{Radial extent}

Figure 13 shows the radial displacement profile of an $n=1$ precursor, together with the position of the pedestal shoulder,

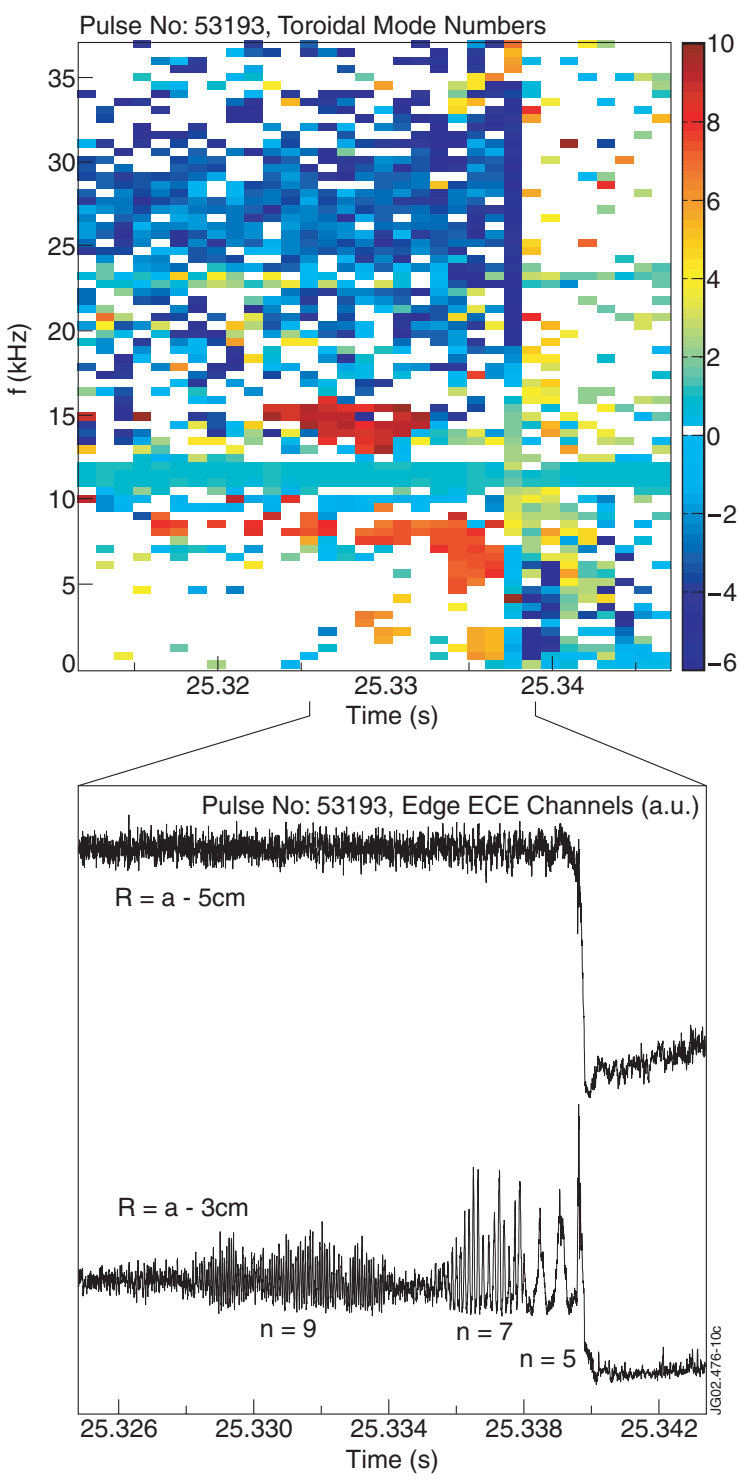

Figure 10. Toroidal mode numbers and ECE emission $5 \mathrm{~cm}$ (near the pedestal top) and $3 \mathrm{~cm}$ (region of steep gradients) inside the separatrix, respectively, showing a rapid change of dominant precursors. The continuous mode at $12 \mathrm{kHz}$ is a sawtooth precursor.

obtained with the same procedure as for the $n=8$ precursor in figure 6 . The remarkable difference compared to the $n=8$ precursor is that the mode width of the $n=1$ precursor does not extend as far as the pedestal shoulder, or even further into the plasma, but that the large displacements only occur within the pedestal radii, that is, further outwards. This could be due to the limited resolution of the ECE diagnostic, but since this pattern occurred systematically in several other cases, it seems that low- $n$ modes have a narrower extent with respect to the width of the transport barrier compared to higher- $n$ modes.

\section{3. $D_{\alpha}$ rise}

The characteristic rise in the level of $\mathrm{D}_{\alpha}$ emission has often been used in the past as a characteristic feature to identify the occurrence of OMs. This $\mathrm{D}_{\alpha}$ rise is qualitatively similar to that observed for higher- $n$ precursors (already shown in figure 8). 


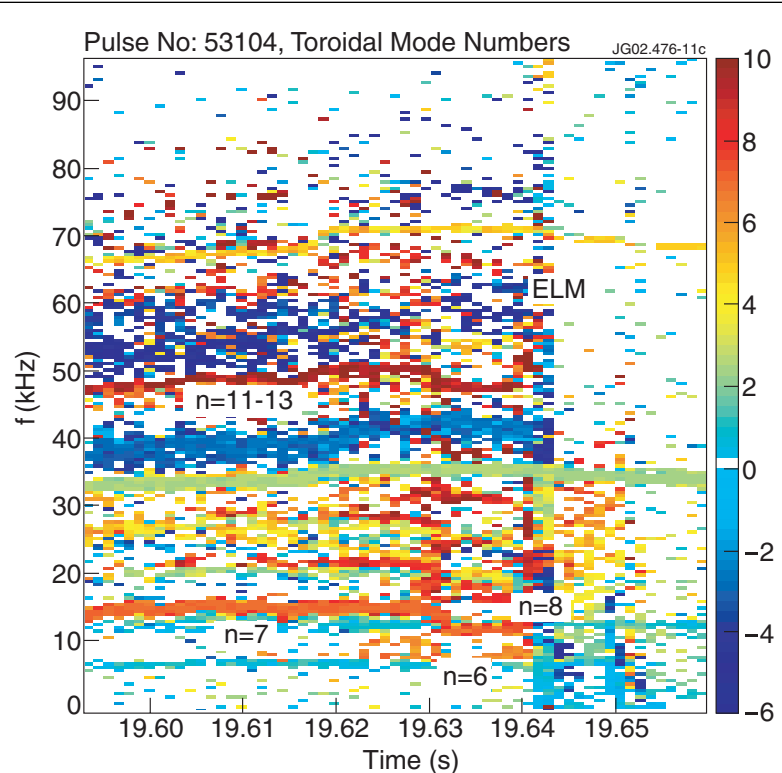

Figure 11. Toroidal mode numbers for an example showing multiple precursors. Also visible is a continuous $4 / 3$ mode around $34 \mathrm{kHz}$ triggered at an earlier time.

\subsection{Ballooning character}

Figure 14 shows the Fourier spectra of signals from a low-field side and a high-field side Mirnov coil for the case of a low- $n$ precursor $(a)$ and a high- $n$ precursor $(b)$. For each discharge the distance of the two coils to the separatrix on the low- and high-field sides is comparable ( $\sim 16 \mathrm{~cm}$ in $(a), \sim 21 \mathrm{~cm}$ in $(b)$ )). For the main component of the $n=1$ precursor the ratio of measured inboard/outboard amplitudes is small, about 1.75. For the $n=8$ precursor this ratio is very high, roughly 30 . This initially suggested that precursors with low- $n$ equal to 1 or 2 have no strong ballooning character, as is expected for external kinks, while with increasing $n$-numbers the ballooning character of the modes increases. There is, however, an effect that needs to be considered in interpreting the data and which imposes an artificial inboard/outboard asymmetry of the magnetically measured signal amplitudes even for modes that do not have ballooning character.

In the cylindrical approximation the mode-induced magnetic fluctuations radially decay with $\left(r_{\text {mode }} / r_{\text {coil }}\right)^{m}$, where $m$ is the poloidal mode number of the mode. Within this approximation, the radial decay of the fluctuations is, therefore, equally strong on the low- and high-field sides. If the mode itself does not have a ballooning character, then coils on the low- and high-field sides pick up fluctuations of equal amplitude. In toroidal geometry, however, the poloidal structure of modes is distorted compared to the cylindrical case. The local poloidal wavenumber $k_{\theta}$ of the mode is higher on the high-field side than on the low-field side (e.g. tearing mode's O- and X-points are not distributed poloidally equidistant, but closer to each other on the high-field side). The result is that the mode-induced magnetic fluctuations on the high-field side are subject to a stronger radial decay due to the higher 'local $m$ ' compared to the fluctuations on the lowfield side. The differing radial decay unavoidably introduces an artificial inboard/outboard asymmetry of the magnetically measured mode amplitudes.
Pulse No: 42840, Mirnov Signal (a.u.)

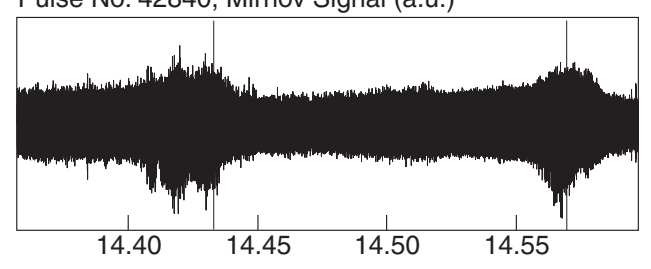

Pulse No: 42840, Magnetics Spectrogram
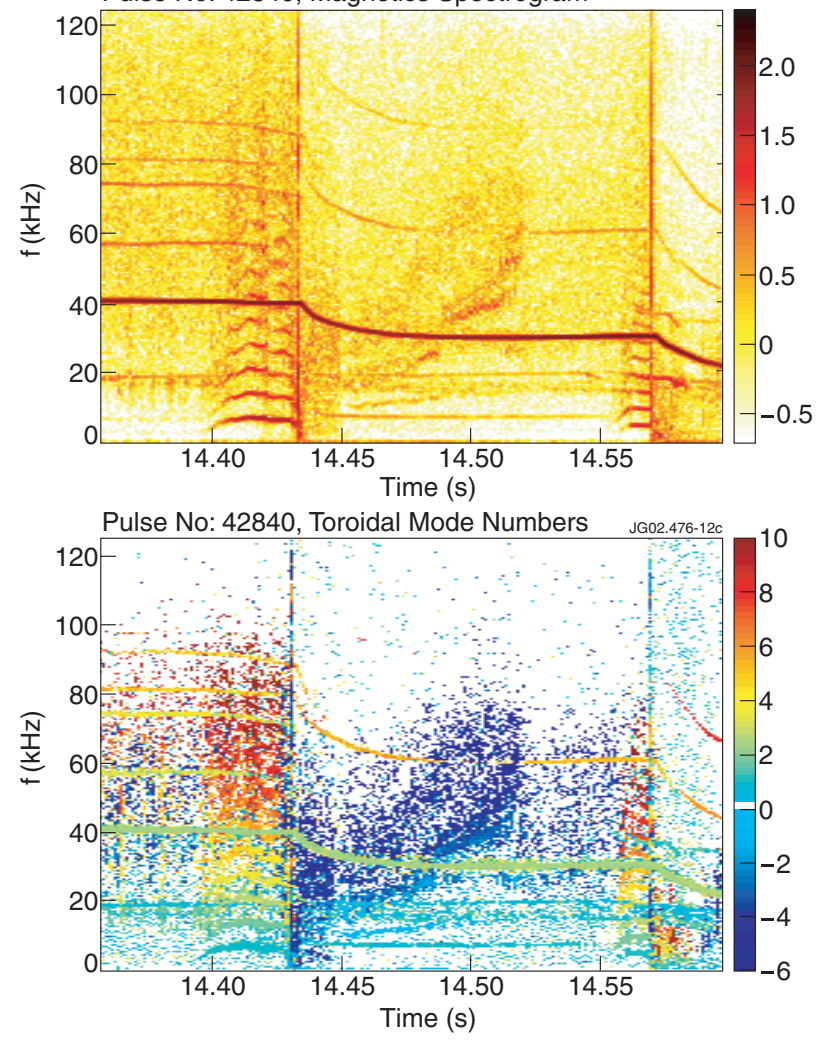

Figure 12. Mirnov signal of a low-field side coil, the corresponding spectrogram, and the spectrum of toroidal mode numbers showing two external kinks. The continuous mode with initially $40 \mathrm{kHz}$ is a $4 / 3$ mode. Like the higher- $n$ precursors, external kinks propagate in the direction of the ion diamagnetic drift.

For modes with low $m$-numbers the effect is not important because the ratio $\left(r_{\text {mode }} / r_{\text {coil }}\right)$ is usually close to one in JET. This is further corroborated by the behaviour of the harmonics of the $n=1$ precursor in figure 14(a). Although the lower- $n$ components show nearly no difference in amplitude on the low- and high-field sides, the difference in inboard/outboard amplitudes gradually increases with the mode numbers of the harmonics, and becomes very pronounced for the higher harmonics.

The effect makes it very difficult to check for the ballooning character of modes through direct comparison of low- and high-field side Mirnov signals. A rough estimate of the effect can be made by following a technique similar to that used in [26]. One assumes a field aligned perturbation with $\vec{k} \cdot \vec{B}=0$, and obtains for the ratio of poloidal wavenumbers of a mode the following expression

$$
\frac{k_{\theta_{2}}}{k_{\theta_{1}}}=\frac{B_{\theta_{1}}}{B_{\theta_{2}}}\left(\frac{R_{1}}{R_{2}}\right)^{2}
$$




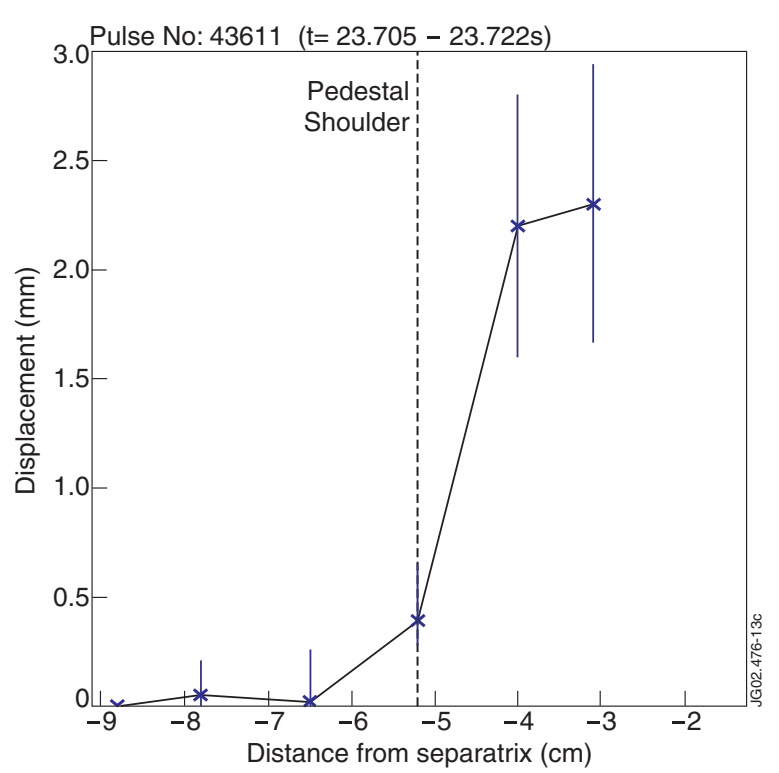

Figure 13. Radial displacement profile of an $n=1$ precursor together with position of the pedestal shoulder. Relative to the width of the transport barrier, lower- $n$ modes are narrower than higher- $n$ modes.

Using EFIT the ratio of wavenumbers on the high/low-field side of $\sim 3.4$ is estimated for the case of figure $14(b)$, which, for high enough $m$, can indeed lead to signal amplitude asymmetries comparable to the ones measured.

In order to verify the above considerations, modelling calculations have been performed with the MISHKA code [27]. The calculations assess the MHD mode propagation in the vacuum region using real JET geometry, and taking into account the coordinates of the Mirnov coils. The JET vessel is considered to be ideally conducting, and the discharge shape is obtained from EFIT. Low- and high- $n$ cases have been studied. The poloidal distortion of the modes is found to cause different radial decays on the low- and high-field sides which agrees very well, quantitatively, with the above considerations.

So, in contrast to what has been presumed in [28], and lacking an accurate enough estimate of the poloidal mode number $m$ of the precursors, it has to be concluded that the magnetic data do not clarify whether the high- $n$ modes really have a ballooning character or not. The observed difference in inboard/outboard signal amplitudes could well originate from the effect discussed above.

It has to be emphasized that the above considerations are of general relevance, and should be taken into account whenever magnetic fluctuations picked up on the low- and high-field sides need to be compared.

\subsection{Parametric studies}

Initial studies to determine parametric dependences of the precursor mode numbers on local quantities have been performed. The precursor mode numbers are found to be mainly prescribed by the edge densities and temperatures. Figure 15(a) shows their distribution in the $n_{e}-T_{e}$ space. The line-averaged electron density has been measured through interferometry using a vertical cord that views the plasma
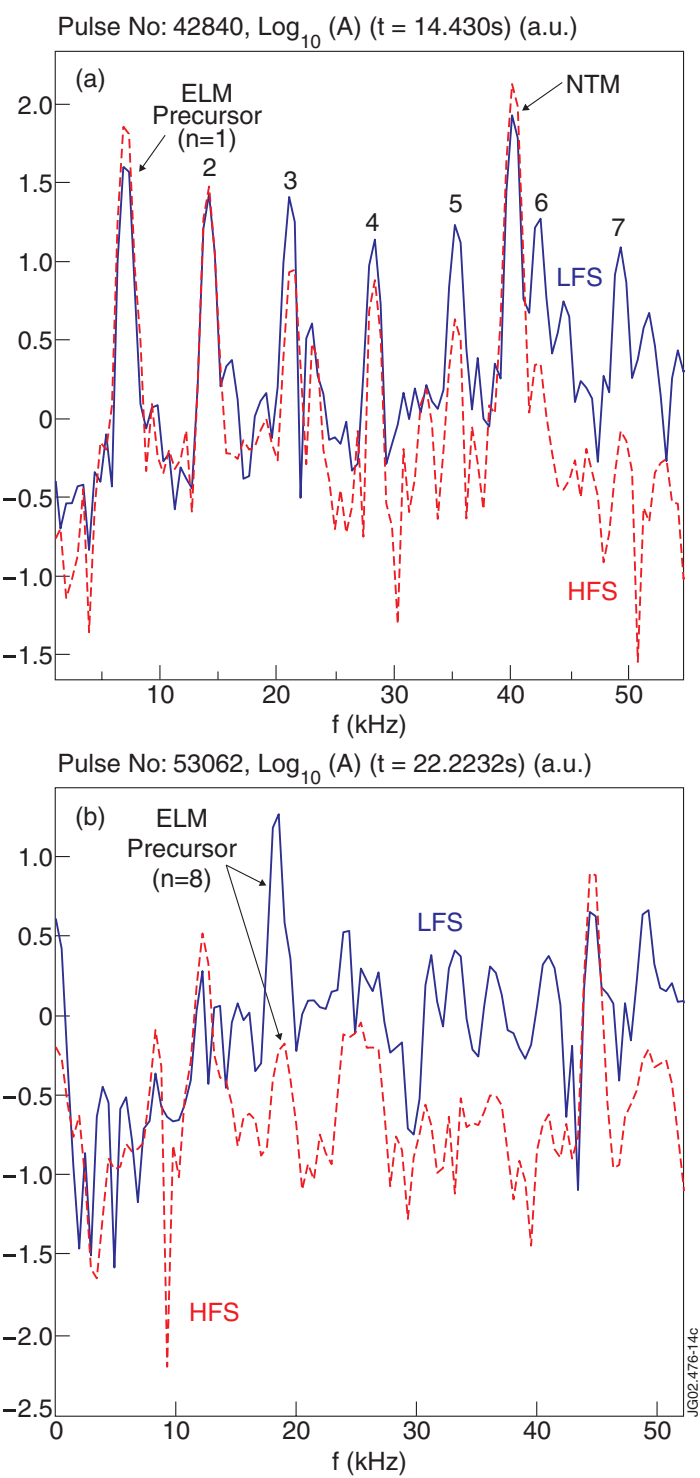

Figure 14. Logarithmic Fourier spectra of signals of a low- and high-field side Mirnov coil with comparable distance to the separatrix $(a$ ) for one of the $n=1$ precursors of figure 12 (where the numbers given denote the $n$-numbers of the harmonics of the precursor), and ( $b$ ) for one of the $n=8$ precursors of figure 2 . For the $n=8$ precursor the ratio of measured amplitudes on the lowand high-field sides is roughly 30 , while for the fundamental component of the $n=1$ precursor this ratio is only $\sim 1.75$. In spite of this low asymmetry, for the harmonics of the $n=1$ precursor the measured inboard/outboard amplitude asymmetry gradually increases with increasing mode numbers of the harmonics.

edge on the low-field side, while the value for $T_{e}$ is taken at the pedestal shoulder and measured via ECE. For the study of parametric scalings, data from 56 discharges have been used. The discharges cover a wide range in parameter space, with $B=2.4-3.9 \mathrm{~T}, I=2.0-4.0 \mathrm{MA}, q_{95}=2.85-5.2$, $\delta=0.22-0.47$, and $P_{\mathrm{NBI}}=8-17 \mathrm{MW}$ (sometimes also combined with ICRH). Each point in the plot corresponds to an ELM precursor. If several precursors occurred prior to an ELM, the dominant one has been selected, while in cases with a fast succession of precursors the first one has been taken. (This convention has been adopted to exclude possible nonlinear effects arising from the preceding precursor.) 

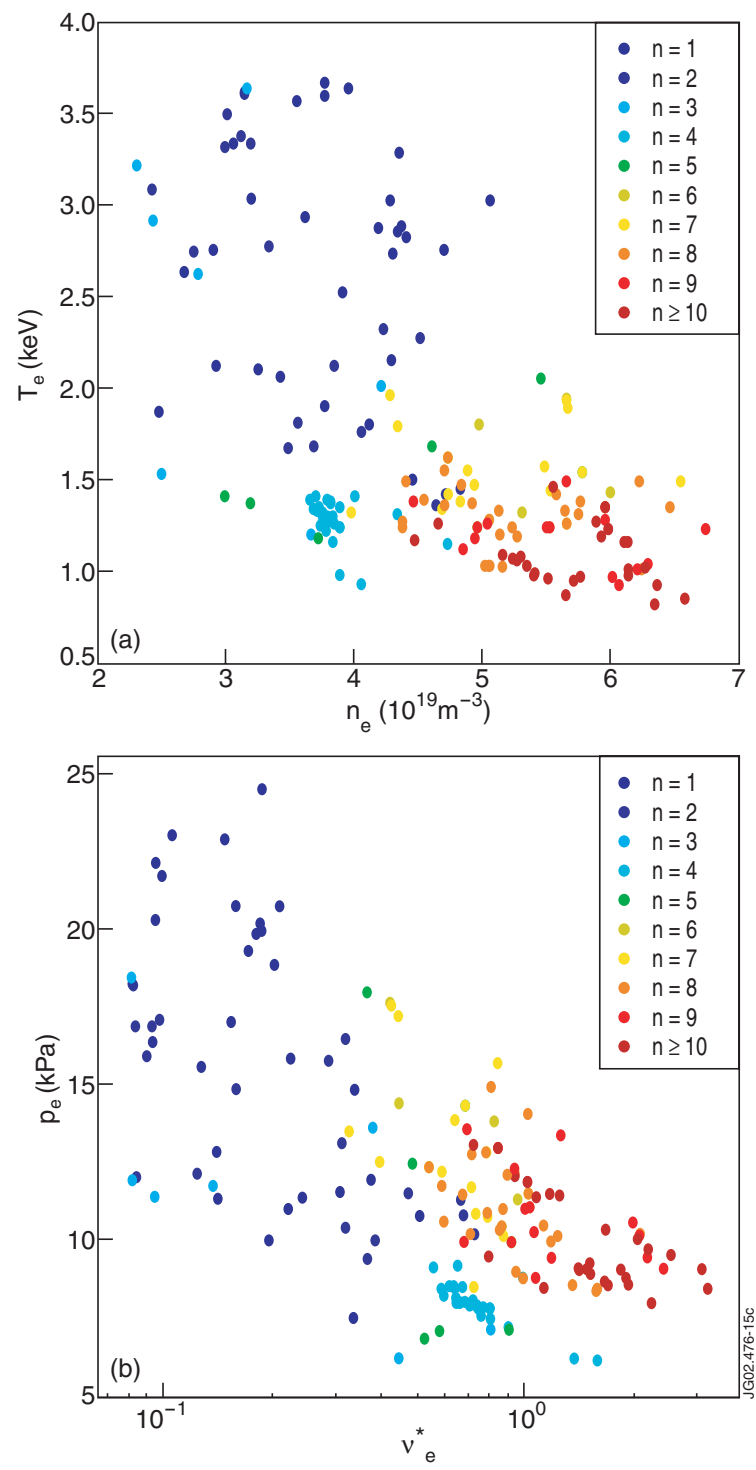

Figure 15. Precursor $n$-numbers as a function of ( $a$ ) electron temperature near the pedestal top and edge line-averaged density, and $(b)$ electron pedestal pressure and normalized electron pedestal collisionality. The data shown include precursors of $1-150 \mathrm{~ms}$ in duration.

Alternatively, one may plot the mode numbers versus the pedestal pressure and the (normalized) electron collisionality $v_{\mathrm{e}}^{*}($ figure $15(b))$, where $v_{\mathrm{e}}^{*}=0.012 n_{\mathrm{e} 20} Z_{\mathrm{eff}} q_{95} R /\left(\epsilon^{3 / 2} T_{\mathrm{ekeV}}^{2}\right)$, taking $Z_{\mathrm{eff}}=2$. $v_{e}^{*}$ determines the amount of bootstrap current for given pedestal gradients [29], with increasing collisionality leading to reduced bootstrap current. Naturally, it would have been desirable to plot the distribution of mode numbers directly as a function of $p_{\mathrm{e}}^{\prime}$ and edge current density, but these quantities could not be measured due to diagnostic limitations.

In figure 15, the pedestal pressure decreases with increasing density (collisionality). This behaviour of the pedestal is consistent with earlier results from density scans on JET [30,31]. It is presumably related to a decrease in the width of the pedestal (keeping the pedestal gradient fixed) with decreasing edge ion temperature [31]. Such a narrowing is further predicted by a variety of scalings for the pedestal width (see, e.g. [32]). Thus, in order to increase the edge current density keeping the pressure gradient fixed, one presumably has to move roughly diagonally in figure $15(b)$, from the lower right corner towards the upper left corner. On the other hand, to increase the edge pressure gradient keeping the edge current density fixed, it is necessary to simultaneously raise the collisionality. This corresponds to a diagonal movement from the lower left corner towards the upper right corner in the plot.

The data in figure $15(b)$ suggest that the mode numbers are determined by a combination of the edge pressure gradient and the edge current driving force. Decreasing the collisionality (increasing the edge current) keeping the pressure gradient fixed, leads to lower mode numbers, while increasing the pressure gradient keeping the edge current fixed raises the mode numbers. In both cases, the transition from low to high $n$-numbers occurs in a relatively narrow region of parameter space. For low enough collisionality (high enough current) only low mode numbers were observed, independent of the pressure. On the other hand, in discharges with sufficiently high edge collisionality (roughly $v_{e}^{*}>1-3$, depending on the discharge configuration) the precursors seem to be absent, indicating that a sufficiently high edge current is necessary to destabilize the precursors. The observed parameter dependence cannot be explained by considering only pure external kinks/peeling modes. Further types of instabilities need to be included.

It is worth comparing the experimentally observed parameter dependence with theoretical predictions. Some recent work has been performed on the exploration of the relationship between the calculated unstable toroidal mode number of edge instabilities and the edge density, edge collisionality and bootstrap current [33-35]. The ELITE code [36], in conjunction with the low- $n$ stability code GATO [37], has been used. The work highlights the importance of the edge current density for the edge MHD stability. In [33], the inclusion of the current in studies of the pedestal leads to a separate dependence of MHD stability on density and temperature, rather than just pressure, because of the strong collisional dependence of the bootstrap current. This is in agreement with the experimental results presented here. The stability calculations have shown that pedestal currents play a dual role in the MHD stability, stabilizing high toroidal mode number ballooning modes through reduction of the edge magnetic shear, while at the same time providing drive for intermediate- to low- $n$ peeling modes. The result is that coupled peeling-ballooning modes at intermediate- $n$ are often the limiting instability. Similar trends have been obtained in $[34,35]$. In [34], a decrease in bootstrap current leads to an increase in the $n$-numbers of the unstable coupled peelingballooning modes. In [35], a set of discharge equilibria with varying density is studied. At low density, the edge bootstrap current stabilizes the higher- $n$ peeling-ballooning modes, which allows the lower- $n$ modes to dominate the instability. At high density, and collisionality, the higher- $n$ modes are no longer stabilized and become dominant before the lower- $n$ modes can grow. These trends are consistent with the experimentally observed collisionality dependence in figure $15(b)$.

The pressure-current dependence indicated by the experimental data might open up the possibility of changing the precursor mode numbers actively by in-shot current ramps. 
Furthermore, it is worth noting that the onset of OMs could be delayed through a slow current ramp down, thus decreasing the edge current, once the H-mode phase is established [38].

The data shown in figure 15 include precursors of $1-150 \mathrm{~ms}$ in duration. A separate treatment of short-lived $(<5 \mathrm{~ms})$ and long-lived ( $>5 \mathrm{~ms}$ ) precursors yielded no evident difference in their respective edge density, edge temperature and edge collisionality. Hence, the time by which the precursor onset precedes the ELM does not seem to be related to those parameters.

To date, no evident dependence of the precursor mode numbers on the plasma triangularity could be identified. Further parametric studies are anticipated.

\section{Modelling results}

To gain insight into the stability limits of the plasma boundary in the H-mode regime in JET, extensive numerical simulations have been performed. As a starting point for the calculations, the JETTO code is used [39], whose equilibrium module takes into account the $\mathrm{H}$-mode transport barrier. It calculates the edge magnetic surfaces self-consistently with the edge pressure gradient and the edge current density, including the bootstrap current contribution. The formulae employed to calculate the bootstrap current can be found in [29]. The pedestal width is a free parameter chosen to match the experimentally observed pressure at the pedestal top. Ideal stability limits are then calculated with the HELENA [40] and MISHKA-1 [27] codes by multiplying the edge pressure gradient and the edge current independently by a constant, keeping the total current and energy content of the plasma fixed. Diamagnetic effects and resistivity were not taken into account for the calculations presented here. The higher order (cubic) finite elements used in all codes allow accurate equilibrium/stability calculations from $n=0$ up to very high toroidal mode numbers $(n \leqslant 50)$. For each equilibrium the stability of the $n=1,2,3,4,6,8,10,12, \ldots$ modes have been calculated, considering the JET vacuum vessel to be ideally conducting.

The instabilities found can be classified into three categories: ballooning modes (the most unstable ones having typically high $n,>10$ ), kink (peeling) modes (mostly low $n$, $<6$ ) and ballooning-kink modes (normally with medium $n$-numbers around 5-15). Due to their overlap, $n$-numbers by themselves are, in principle, only of limited use in classifying the modes. The classification relies more on the driving forces and different mode structures as described below. Ballooning modes are driven by the pressure gradient, $\alpha=$ $-\mu_{0} p^{\prime} q^{2} / B_{0}^{2} \epsilon$, and stabilized by shear, whereas kink (peeling) modes are driven by the edge current density and stabilized by pressure. Kink-ballooning modes are driven by both the pressure gradient and the edge current density.

Figure 16 shows the calculations for an ELMy H-mode discharge, where the stability limits for the different mode types are displayed. The coloured areas show the stability limits of infinite- $n$ ballooning modes calculated by HELENA at different values of the normalized poloidal flux function near the edge ( $\psi=0.95-0.99)$. The numbers displayed in the plot have been calculated by MISHKA, and represent the $n$-numbers of the most unstable finite- $n$ modes up to $n=14$ at the respective $\alpha$ and edge current values. For sufficiently

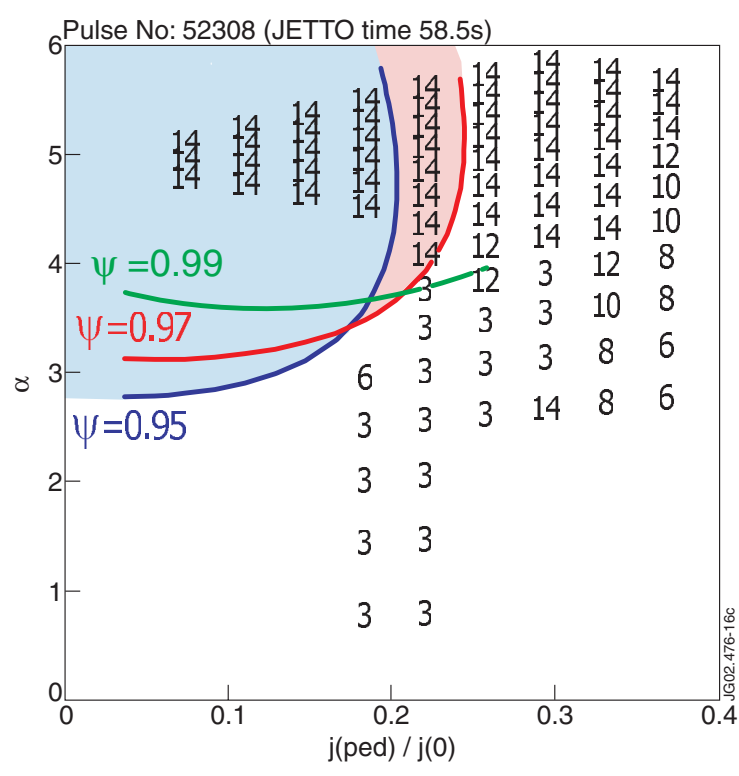

Figure 16. Stability limits for $n=1-14$ modes (numbers) and for $n=\infty$ ballooning modes (coloured areas) for an ELMy-H discharge, as a function of the ballooning parameter $\alpha$ and the normalized edge current density.

low values of the edge current density and $\alpha$, i.e. in the area in the lower left corner in the plot where no mode numbers are indicated, the plasma edge is found to be stable. For the unnumbered region in the lower right corner of the plot, well inside the kink stability limit, the stability of points has not been further investigated (modes becoming unstable far beyond the instability boundaries are not likely to be encountered in the experiment any more)

While in figure 16 there is some scattering in the distribution of the most unstable mode numbers, the general trend that is reproducibly encountered in the discharges analysed is to obtain high $n$-numbers at high $\alpha$, where the ballooning modes are expected, while at low- $\alpha$ and finite edge current low- $n$ modes become destabilized. These correspond to kink modes. Intermediate- $n$ modes are often encountered in the proximity of both the kink and the ballooning stability limit, and in most cases belong to coupled peeling-ballooning modes. The variation in mode number near $\alpha=3$ in figure 16 reflects the fact that high- and low- $n$ growth rates become very similar in that region. At higher $\alpha$ values, the difference in growth rate between low- and high- $n$ modes increases again.

A direct comparison of figures $15(b)$ and 16 is not valid. Figure $15(b)$ plots mode numbers as a function of $p_{e}$, not the pressure gradient. As has been discussed in the previous section, the pedestal width is expected to increase with increasing edge ion temperature.

For comparison with experiment only the mode numbers close to the marginal stability boundaries are relevant. The experimentally observed precursor mode number will depend on the trajectory of the discharge in $\alpha$ and in pedestal current, and where it first crosses an instability boundary. Low edge collisionality will favour high edge current density relative to the magnitude of the edge pressure gradient, and hence the discharge will cross the marginal stability boundary at the kink limit, where low $n$-numbers are predicted. Accordingly, high edge collisionality will favour higher $n$-numbers. This is 

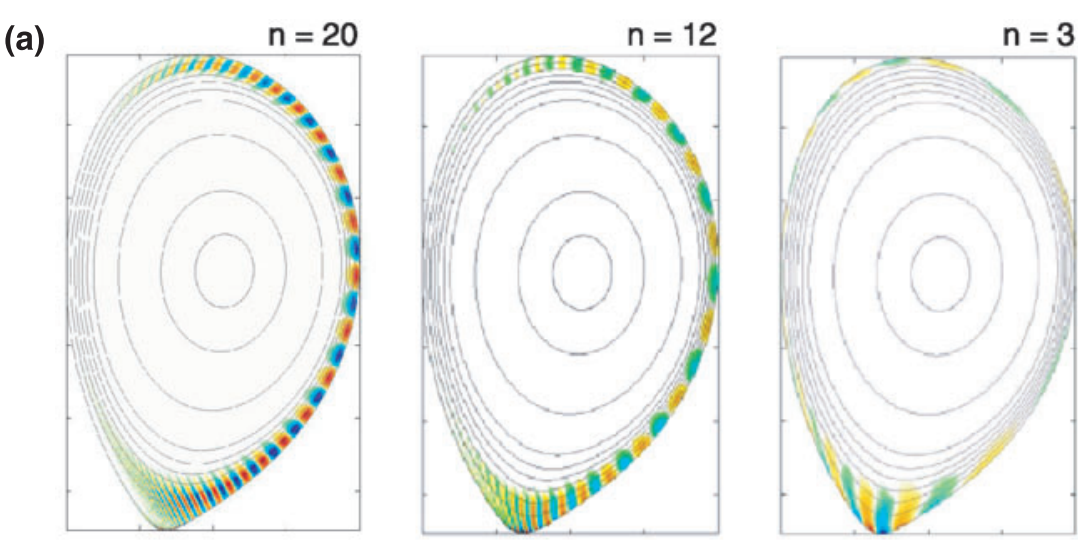

(b)

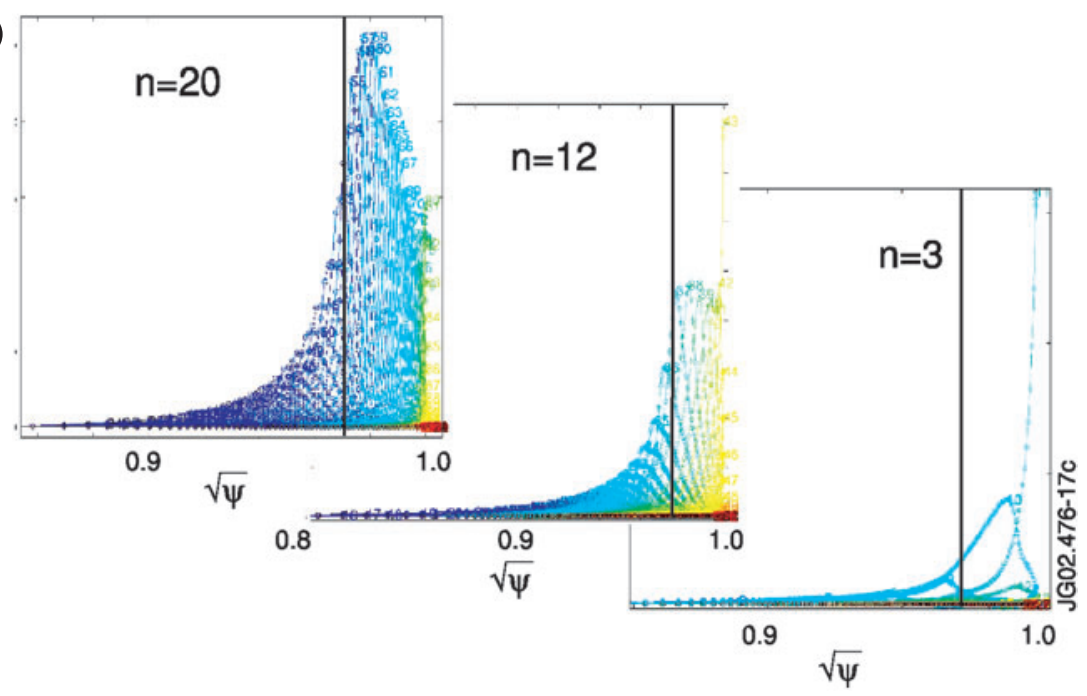

Figure 17. Poloidal structure of the radial displacements of an $n=20$ ballooning, an $n=12$ coupled ballooning-kink and an $n=3$ kink instability together with their midplane radial displacement profiles. Each curve corresponds to a poloidal component with its respective poloidal mode number $m$. The vertical lines denote the position of the pedestal shoulder.

again in qualitative agreement with figure 15(b). Quantitative comparisons of modelling predictions with the mode numbers observed in the experiment are difficult due to limitations in the determination of edge quantities such as the edge current density. They would further require detailed computations for each different case, which is beyond the scope of this paper. Here, the aim is to make qualitative comparisons between theory and the observed precursors.

As mentioned above, the mode structures of the three types of instability differ significantly. Figure 17(a) shows plasma cross sections with the poloidal mode structures of an $n=20$ ballooning, an $n=12$ ballooning-kink and an $n=3$ kink instability, respectively. The ballooning mode shows the expected large asymmetry of the mode amplitude on the high- and low-field sides, and has a relatively broad radial extent. The $n=3$ kink has a comparable inboard/outboard mode structure, a much narrower extent, particularly around the midplane, and shows, in contrast to the ballooning mode, large and broad mode displacements concentrated around the $\mathrm{X}$-point. The ballooning-kink mode shows a mixture of these properties, with a much larger amplitude on the low-field side than on the high-field side, broad radial mode extent and distinct displacements around the X-point.
Also, in figure 17, the respective midplane radial displacement profiles are shown. The vertical line around $\sqrt{\psi}=0.97$ denotes the position of the pedestal shoulder, which is held invariant as the edge current and pressure are scanned. Thus, the extent of ballooning and ballooning-kink modes is comparable to the width of the transport barrier, while the $n=3 \mathrm{kink}$ is narrower. This is consistent with the experimental results presented in section 3 . The kink-mode displacement increases sharply towards the separatrix, while the ballooning displacement peaks well inside it. Ballooning mode peak amplitudes occur at the position of maximum pressure gradient. Both ballooning and ballooning-kink modes are composed of several $m$-components, whereas the kink mode shows only a small number of them.

As described in section 3, the experimental measurements confirm that lower- $n$ precursors have a narrower radial extent compared to the higher- $n$ precursors. This is consistent with the lower- $n$ precursors being external kinks, and also suggests that the higher- $n$ precursors are not pure external kinks but, according to their mode width, either peeling-ballooning or pure ballooning modes. In addition, the observed precursor $n$-numbers are mostly below $n=10$, which according to modelling is rather too low for pure ideal ballooning 
modes. Hence, modelling results are consistent with the low- $n$ precursors being ideal external kinks, while higher- $n$ precursors are most likely ideal peeling-ballooning modes.

\section{Summary and discussion}

The properties of a class of type-I ELM precursors, which is commonly observed in JET in ELMy-H discharges with co-injected NBI, have been studied in considerable detail. Their characteristic features comprise: low frequency, mostly below $25 \mathrm{kHz}$; typical onset a few milliseconds to a few tens of milliseconds before the ELM crash; propagation in the direction of the ion diamagnetic drift; localization in the pedestal region extending to the separatrix or beyond with no radial phase inversion; and a slight increase of the $\mathrm{D}_{\alpha}$-emission accompanying their occurrence. Radial mode displacements from $1 \mathrm{~mm}$ up to $\sim 1.5 \mathrm{~cm}$ have been observed.

Due to the closeness of rational surfaces near the separatrix combined with the short scale length on which quantities like pressure gradient, edge current density, plasma rotation or influence of neutrals change radially, one can expect each mode to have a narrow instability window that may be easily disturbed. The observations made of multiple precursors at similar radii, sometimes changing their toroidal mode numbers and frequency with no obvious reason, suggest that this is indeed the case.

A range of mode numbers $n=1-13$ has been observed, with the low- $n$ precursors known to be ideal external kinks. A thorough comparison of low- and high- $n$ precursors has been made both in experiment and through modelling calculations and suggests the moderate- $n$ precursors are coupled peelingballooning modes (and not pure ballooning modes, which occur at higher- $n$ ). Pure peeling modes are precluded by the observation of the mode width, which is too broad for peeling modes, and because of the observed dependence of mode number on $n_{e}$ and $T_{e}$ (cf figure 15). It rather seems that the precursor $n$-numbers are determined by some interplay of both the pressure gradient and the edge current density. Due to the absence of radial phase inversions, tearing modes have to be excluded.

If the precursors are involved at all in the ELM cycle, they do not seem to be the actual trigger for the ELM. This is concluded from the observation that the precursor oscillations do not grow exponentially into the ELM crash but mostly rather linearly. In fact, there is no evidence of the precursor mode growth rate rapidly accelerating before the ELM, and cases were shown where the precursor amplitude even remains nearly saturated. A further point that suggests that the ELM precursors do not trigger the ELMs has been previously given in [38]. The technique of current rampdown used to optimize the performance of hot-ion $\mathrm{H}$-mode discharges was found to delay the onset of the low- $n$ precursors (OMs) while making the ELMs appear earlier. ELMs were still preceded by the OMs in the current rampdown discharges, but the duration of the OMs was reduced compared to the discharges without current rampdown. This implies that the ELM trigger behaved differently to the OMs during the current rampdown, indicating that the ELM precursor and ELM trigger are two separate phenomena. This and the large variation in the time by which the precursor appearance precedes the ELM could be explained in terms of the ELM event resulting from a combination of two instabilities. Within this picture a precursor mode starts once the stability limit for the first instability is encountered, but the ELM is not triggered until the stability limit for the second instability is reached. The model of the peeling-ballooning cycle for type-I ELMs [41] points very much in that direction. However, if one assumes ELMs to be finally triggered by a combination of kink- and ideal ballooning modes, at least in the particular case of discharges with low- $n$ precursors the kinklimit is obviously reached before the ideal ballooning limit. This is in disagreement with the order of events predicted in the peeling-ballooning cycle originally proposed in [41]. A generalization of the peeling-ballooning model with ELM cycles for which the kink/peeling boundary is encountered first has been recently proposed in [33].

On the other hand, the precursors seem to be absent in discharges with sufficiently high electron edge collisionality (strong gas puffed discharges) for which $v_{e}^{*}>1-3$ (depending on the discharge configuration), and also optimized shear discharges were reported where they could not be seen [42], raising the question as to whether the precursors are really linked to the ELM event. The absence of precursors in high collisionality discharges is not believed to be related to a diagnostic limitation. Although in many of these cases the ECE data are not available (making it difficult to find short-lived precursors, in particular), sufficiently long-lived precursors should still be detectable through the magnetics. However, they have not been detected in spite of the considerable number of high density discharges analysed. This is in contrast to what is observed in discharges with low to moderate collisionality. Gas scans of discharges have been analysed in which the precursors still had relatively low $n$, equal to 5-6, for a number of discharges at moderate fuelling rates, prior to their disappearance at the next highest density. The lack of precursors at high collisionality (low edge current) would again be consistent with their identity as ideal kinkor coupled ballooning-kink modes, and not ideal ballooning modes. A hypothesis that is supported by the overall data presented here is that the ideal ballooning modes, and not ideal kink- or coupled ballooning-kink modes, could provide the trigger for type-I ELMs. In this respect, certain theoretical studies $[43,44]$ predict a general explosive feature of the ideal ballooning instability ('detonation') emerging from nonlinear effects through the development of fine-scale substructures ('fingers'). In any case, the importance of the ELM precursors studied here lies in the fact that they provide a valuable source of information to improve the understanding of ELM-cycles, and their further study certainly opens the possibility to validate, or refute, present ELM models.

The precursor phenomenon does not seem to be restricted to JET. The precursors show similarities with the ones reported in $[6-8,14]$, particularly their low frequency and typical duration in the milliseconds range, as well as the mode numbers reported in $[6,7]$.

A significant feature of ELMy H-modes in JET is the universally observed washboard modes [20]. The properties of these modes, which seem to significantly interact with the ELM precursors, will be discussed in [21]. 


\section{Acknowledgments}

This work has been performed under the European Fusion Development Agreement. C.P.P. would like to acknowledge the financial support of the Dutch Research Organisation, NWO.

\section{References}

[1] Zohm H. 1996 Plasma Phys. Control. Fusion 38105

[2] Connor J.W. 1998 Plasma Phys. Control. Fusion 40191

[3] Connor J.W. 1998 Plasma Phys. Control. Fusion 40531

[4] Leonard A.W. et al 1999 J. Nucl. Mater. 266-269 109

[5] Janeschitz G. 2001 J. Nucl. Mater. 290-293 1

[6] Suttrop W. et al 1996 Plasma Phys. Control. Fusion 381407

[7] Kass T. et al 1997 Proc. 24th EPS Conf. on Controlled Fusion and Plasma Physics (Berchtesgaden) vol 21A (part IV) ed M. Schittenhelm et al (Geneva: EPS) p 1521

[8] Manso M. et al 1998 Plasma Phys. Control. Fusion 40747

[9] Maraschek M. et al 1998 Proc. 25th EPS Conf. on Controlled Fusion and Plasma Physics (Praha) vol 22C, p 492

[10] Buttery R.J. et al 1995 Proc. 21st EPS Conf. on Controlled Fusion and Plasma Physics (Bournemouth) vol 19C (part III) p 273

[11] Colton A.L. et al 1996 Plasma Phys. Control. Fusion 381359

[12] Zohm H. et al 1995 Nucl. Fusion 35543

[13] Doyle E.J. et al 1991 Phys. Fluids B 32300

[14] Oyama N. et al 2001 Plasma Phys. Control. Fusion 43717

[15] Nave M.F.F. et al 1995 Int. Conf. on Plasma Physics ICPP 1994 (Foz do Iguacu, Brazil, 1994) (American Institute of Physics) AIP Conf. Proc. 34558

[16] Ali-Arshad S. et al 1992 Proc. 19th EPS Conf. on Controlled Fusion and Plasma Physics (Innsbruck) vol 16C (part I) p 227

[17] JET Team (presented by Jones T.T.C.) 1995 Plasma Phys. Control. Fusion 37 A359

[18] Nave M.F.F. et al 1997 Nucl. Fusion 37809

[19] Huysmans G.T.A., Hender T. and Alper B. 1998 Nucl. Fusion 38179
[20] Smeulders P. et al 1999 Plasma Phys. Control. Fusion 41 1303

[21] Perez C.P. et al 2004 Plasma Phys. Control. Fusion 4661

[22] Merezhkin V.G. 1978 Sov. J. Plasma Phys. 4152

[23] Klueber O. et al 1991 Nucl. Fusion 31907

[24] Buttery R.J. et al 2003 Nucl. Fusion 4369

[25] Bornatici M., Cano R., de Barbieri O. and Engelmann F. 1983 Nucl. Fusion 231153

[26] Snipes J.A. et al 2002 Proc. 29th EPS Conf. on Controlled Fusion and Plasma Physics (Montreux) P1.057

[27] Mikhailovskii A.B. et al 1997 Plasma Phys. Rep. 23844

[28] Perez C.P. et al 2002 Proc. 29th EPS Conf. on Controlled Fusion and Plasma Physics (Montreux) P1.023

[29] Sauter O. and Angioni C. 1999 Phys. Plasmas 62834

[30] Saibene G. et al 2002 Plasma Phys. Control. Fusion 441769

[31] Saibene G. et al 1999 Nucl. Fusion 391133

[32] Sugihara M., Takizuka T. and International H-Mode Edge Pedestal Expert Group 2002 Plasma Phys. Control. Fusion 44 A299

[33] Snyder P.B. et al 2002 Phys. Plasmas 92037

[34] Mossessian D.A. et al 2002 Plasma Phys. Control. Fusion 44423

[35] Leonard A.W. et al 2003 Phys. Plasmas 101765

[36] Wilson H.R., Snyder P.B., Huysmans G.T.A. and Miller R.L. 2002 Phys. Plasmas 91277

[37] Bernard L.C., Helton F.J. and Moore R.W. 1981 Comput. Phys. Commun. 24377

[38] Nave M.F.F. et al 1999 Nucl. Fusion 391567

[39] Cenacchi G. and Taroni A. 1988 Rapporto ENEA RT/T1B $88(5)$

[40] Huysmans G.T.A. et al 1991 Proc. CP90 Conf. on Comp. Physics ed A. Tanner (Singapore: World Scientific) p 371

[41] Connor J.W., Hastie R.J. and Wilson H.R. 1998 Phys. Plasmas 52687

[42] Alper B. et al 2002 Proc. 29th EPS Conf. on Controlled Fusion and Plasma Physics (Montreux) P1.025

[43] Hurricane O.A., Fong B.H. and Cowley S.C. 1997 Phys. Plasmas 43565

[44] Fong B.H. 1999 Metastable and explosive properties of ballooning modes in laboratory and space plasmas $P h D$ Thesis University of Princeton, available at http://www.asp.ucar.edu/ bhlfong/thesis.pdf 